\title{
Spectral Engineering via Complex Patterns of Circular Nano-object Miniarrays: I. Convex Patterns Tunable by Integrated Lithography Realized by Circularly Polarized Light
}

\author{
Áron Sipos ${ }^{1} \cdot$ Emese Tóth $^{2}$ • Olivér A. Fekete ${ }^{2} \cdot$ Mária Csete $^{2}$ (I)
}

Received: 8 April 2020 / Accepted: 15 July 2020 / Published online: 16 November 2020

(C) The Author(s) 2020

\begin{abstract}
Illumination of colloid sphere monolayers by circularly polarized beams enables the fabrication of concave patterns composed of circular nanohole miniarrays that can be transferred into convex metal nano-object patterns via a lift-off procedure. Unique spectral and near-field properties are achievable by controlling the geometry of the central nanoring and quadrumer of slightly rotated satellite nanocrescents and by selecting those azimuthal orientations that promote localized plasmon resonances. The spectral and near-field effects of hexagonal patterns composed of uniform gold nanorings and nanocrescents, which can be prepared by transferring masks fabricated by a perpendicularly and obliquely incident single homogeneous circularly polarized beam, were studied to uncover the supported localized plasmonic modes. Artificial rectangular patterns composed of a singlet nanoring and singlet nanocrescent as well as quadrumer of four nanocrescents were investigated to analyze the effect of nano-object interactions and lattice type. It was proven that all nanophotonical phenomena are governed by the azimuthal orientation independent localized resonance on the nanorings and by the $\mathrm{C} 2, \mathrm{C} 1$, and $\mathrm{U}$ resonances on the nanocrescents in case of $\bar{E}$-field direction perpendicular and parallel to their symmetry axes. The interaction between localized surface plasmon resonances on individual nano-objects is weak, whereas scattered photonic modes have a perturbative role at the Rayleigh anomaly only on the larger periodic rectangular pattern of miniarrays. Considerable fluorescence enhancement of dipolar emitters is achievable at spectral locations promoting the $\mathrm{C}$ and $\mathrm{U}$ resonances on the constituent nano-object.
\end{abstract}

Keywords Spectral engineering - Nanoplasmonics Convex nanoparticle patterns $\cdot$ Localized surface plasmon resonance . Rayleigh anomaly · Fluorescence enhancement of dipolar emitters · Tunable spectral properties · Tunable near-field properties

Electronic supplementary material The online version of this article (https://doi.org/10.1007/s11468-020-01235-2) contains supplementary material, which is available to authorized users.

Mária Csete

mcsete@physx.u-szeged.hu

Áron Sipos

sipos.aron@brc.hu

1 Institute of Biophysics, Biological Research Centre, Szeged, Temesvári krt. 62, H-6726, Hungary

2 Department of Optics and Quantum Electronics, University of Szeged, Szeged, Dóm tér 9, H-6720, Hungary

\section{Introduction}

Investigation of individual and periodic plasmonic structures initialized the inspection of complex patterns composed of metal nano-objects, which have interesting synergetic near-field and spectral properties. The building blocks of these structures are usually uniform nano-objects, and the array optical properties can be tuned by varying the geometrical parameters of the constituent nano-objects and the periodicity of their pattern [1-4].

The optical properties of individual convex scatterers depend on their size and shape and are influenced by the dielectric environment as well $[5,6]$. The extinction 
spectrum of small spherical particles is dominated by a dipolar resonance, while on larger particles, multipolar modes develop as well [7]. Plasmon hybridization on spherical nanoparticles with finite metal walls, like rings and shells, makes it possible to attain a larger number of multipolar modes that are tunable by varying the geometrical parameters [8]. The symmetric (antisymmetric) modes result in peaks, which are red- (blue-) shifted by decreasing the wall thickness to core ratio $[9,10]$.

On dimers (trimers) of core shells, electric (magnetic) dipoles develop, while on heptamer miniarrays, Fanolike peaks appear due to the coexistent bright and dark modes originating from parallel and anti-parallel dipoles [11]. Asymmetric plasmonic trimer structures exhibit a broadband polarization-independent far-field scattering and enhanced near-field simultaneously [12]. The Fano peaks can be precisely tuned on quadrumers and on asymmetric heptamers by the azimuthal orientation, as well as on hetero-octamers by the geometrical parameters [13, 14]. Oligomers built up from a nanocross and a nanorod acts as an ultracompact directional antenna at the Fano resonance, whereas hetero-heptamers consisted of a central nanocross and six satellite rod-pairs result in multiple Fano-lines [15].

It was shown that hexagonal and square patterns composed of hemi-ellipsoids are capable of resulting in band gaps, which can be optimized by tuning the geometrical parameters [16]. On hexagonal and rectangular arrays of cylinders and prisms, dipolar coupling results in a distance-dependent spectral shift [17]. The dispersion characteristics of hexagonal patterns composed of convex nanoparticles are governed mainly by LSPR, in contrast to nanoholes in metal films, where the diffractive coupling on the pattern results in characteristic propagating plasmon-related bands [18]. The diffractive coupling can result in sharp peaks on the extinction of convex patterns, when the lattice period is tuned to the LSPR of individual nanoparticles, which phenomenon is the surface lattice resonance (SLR) [1-3, 19, 20]. The interaction of localized plasmon resonance and diffraction orders results in narrow peaks on the normalized near-field as well [21]. Moreover, adding randomness to rectangular arrays of nanoparticles makes it possible to realize fine spectral engineering and to achieve further enhanced $\bar{E}$-field [22].

For randomly arranged $\mathrm{C}$-shaped gold nanocrescents prepared by shadow sphere lithography (SSL), the polarization, opening angle, thickness, and diameter dependence of the resonances was demonstrated [23]. The $\mathrm{C}$ and $\mathrm{U}$ resonances excited by light polarized perpendicular and parallel to the symmetry axes were identified as antenna-like resonances. The polarization dependence of multipoles, accompanying $\bar{E}$-field confinement and corresponding resonance spectra, was demonstrated on individual C-shaped split-ring resonators (SRRs) as well [24].
For hexagonal arrays of C-shaped resonators created by colloid sphere lithography (CSL), the strong effect of the opening angle as well as of the polarization on the parity and strength of excitable plasmonic modes were demonstrated [25]. On rectangular arrays of gold SRRs, modes of different orders were demonstrated based on the $\bar{E}$-field component along the propagation direction [26]. Caused by the finite scaling regime of magnetic resonances of gold, application of $\mathrm{Al}$ was suggested [27, 28].

Complex plasmonic materials consisted of varieties of SRRs can act as metamaterials as well. Single-ring multi-cut building blocks in rectangular arrays with unit cells in the order of $100 \mathrm{~nm}$ are capable of shifting the operation regions into the visible regime [29]. Cavity resonances excitable on SRR arrays can possess high-quality factors [30]. In case of rectangular patterns of U-shaped SRR doublets, the asymmetry of coupled elements makes it possible to excite dark modes resulting in narrow lines, which is advantageous in sensing [31]. Mirror symmetry broken metamaterials show Fano resonances of controllable width that can be tuned by the temperature [32].

Arrays of various plasmonic objects have been extensively applied in biosensing. Hexagonal patterns composed of nano-tetrahedra and spheroids prepared by colloid sphere lithography have shown biosensing capabilities [6]. Shortrange ordered disks and oblate spheroids prepared by CSL exhibit a sensitivity that depends on the individual object geometry [33]. Three-dimensional nanocrescents are capable of promoting SERS [34]. Via plasmonic nano-diskarrays, six orders of magnitude fluorescence enhancement were observed on pre-designed chips [35]. Two orders of magnitude spontaneous emission enhancement have been achieved in case of dipolar emitters oriented perpendicular to the torus axes that are arranged on gratings [36]. This example indicates that complex patterns composed of predesigned rings can result in considerable enhancement. The highest fluorescence efficiency can be achieved by positioning tapered antennas like spheres, disks, and tori above thin metals films [37]. These results predict that a combination of thin films and spherical antennas is advantageous in sensing of dipolar emitters.

To fabricate complex patterns, various methodologies have already been developed. Via shadow sphere lithography randomly distributed nanocrescents can be fabricated by evaporating metal layers onto surfaces consisted of randomly distributed colloids [23, 24, 34]. Individual colloid spheres or monolayers of them can be used as masks as well. A special methodology has been developed to fabricate randomly distributed nanorings on surfaces, which exhibit spectral peaks in regimes not accessible via disks, moreover support special magnetic states, when made of ferromagnetic materials $[9,38]$. Consecutive 
deposition of silica and gold layers from different polar and azimuthal orientations during shadow sphere lithography allows creating asymmetrical chiral nanocrescents, which are randomly arranged [39]. CSL makes it possible to generate various nano-objects (rings, crescents, cups, rods, and wires); however, all ordered patterns inherit the hexagonal symmetry of the closely packed monolayers [25, 40, 41]. Complex structures with hexagonal symmetry including various building blocks and multiple materials can be fabricated via sequential deposition from multiple angles in CSL [42]. Edge spreading lithography is capable of creating double rings made of gold with a thickness on the order of $10 \mathrm{~nm}[43,44]$. On-edge colloid sphere lithography makes it possible to generate a hexagonal pattern of asymmetrical chiral nanocrescents, by depositing silica and gold from different directions [45].

Special multistep methodologies have been developed to break the hexagonal symmetry and to overcome the monodispersity achievable via colloid sphere monolayers. To fabricate hierarchical structures, CSL was combined with RIE and annealing as well as tilted evaporation, photomasks projection, metal sputtering, and ion milling; the latter is capable of resulting in nanorings [46]. However, in the case of photomask projections, the periodicity of the mask was significantly larger than the colloid sphere diameter in the illuminated monolayer.

Deposition of gold nanoparticles was realized onto periodic patterns created by two-beam interference on colloidal crystals, where photo-switching in the medium promotes chemical attachment [47]. The period of the interference pattern was one and two orders of magnitude larger than the diameter of spheres forming the monolayer. Binary colloid monolayers were created with a characteristic size-scale ratio in the order of $0.19-0.4$; however, the hexagonal symmetry was inherited also in this case [48]. To generate rectangular patterns of nanocrescents and nanorings, only e-beam lithography has been extensively applied previously [26-32].

In this paper, we present complex structures, which are composed of convex miniarrays of a central nanoring and satellite nanocrescents, arrayed in a rectangular pattern. These structures can be fabricated by combining colloid sphere and interference lithography, in a two-steps procedure [49-52]. In our former paper, it was described that the circular nano-objects originate from circularly polarized light, whereas the pattern periodicity is determined by the interference pattern periodicity [51]. The circular nanoobjects originate from the circular lobes in the near-field of metal nanoparticles illuminated by circularly polarized light that were transferred into permanent topology modification of photosensitive azobenzene-dye polymers [53]. Moreover, asymmetrical nanocrescents can be also fabricated without the need for sophisticated lithography [39, 45, 51, 54]. The interferometric illumination of colloid sphere monolayers
(IICSM) enables the direct structuring of positive resists, whereas the masks can be transferred into convex patterns by a lift-off procedure. The spectral and near-field properties of complex concave patterns composed of rounded nanohole miniarrays are described in Part II of this paper [55]. In order to uncover the localized modes supported by the building blocks, the spectral and near-field effects of hexagonal patterns composed of uniform nanorings and nanocrescents that can be fabricated by colloid sphere lithography followed by a lift-off procedure were also studied. To analyze the effect of nano-object interactions and the lattice type in the collective resonance, artificial rectangular patterns of a singlet nanoring and singlet nanocrescent as well as quadrumer of four nanocrescents were investigated. A comparative study on the spectral and near-field effects of concave and convex patterns, which are achievable via IICSM directly and via a consecutive lift-off procedure, is provided as well in our previous work and in an upcoming paper $[52,56]$.

\section{Method}

\section{Numerical Modeling and Characterization of Patterns composed of Convex Spherical Nano-objects}

The finite element method (FEM) implemented into the radio frequency module of COMSOL Multiphysics software package (COMSOL AB, Sweden) was used to determine the geometrical parameters of convex patterns achievable with IICSM. The $\bar{E}$-field distribution was examined under the $\mathrm{Au}$ colloid sphere monolayer on the upper surface of the gold-resist bilayer coated target. In these studies, all materials were modeled by taking into account the wavelength-dependent optical properties [57, 58].

The schematic drawing in Fig. 1a shows the gold-resist bilayer coated target applied for illumination by circularly polarized light, in which the treatment is followed by a liftoff procedure to convert the concave resist hole-pattern into an analogue convex metal pattern. Figure $1 \mathrm{~b}$ and $\mathrm{c}$ show the main characteristic geometrical parameters variable via IICSM methodology, when it is realized by circularly polarized beams. In the closely packed in between arrays illumination configuration the $p^{n}$ pattern periodicity is determined by the $\theta^{n}$ angle of incidence and $\lambda$ wavelength, and it can be tuned in half colloid sphere diameter $\left(n^{*} d / 2\right)$ steps. The $t$ inter-object distance is determined by the $\alpha$ and $\beta$ orientation of the incidence plane and of the interference pattern. The $d_{0}$ and $a$ nano-object size parameters not only are dependent on the wavelength and power density, colloid sphere $d$ diameter, and material, but so can be influenced by the lift-off procedure as well (Fig. 2). 


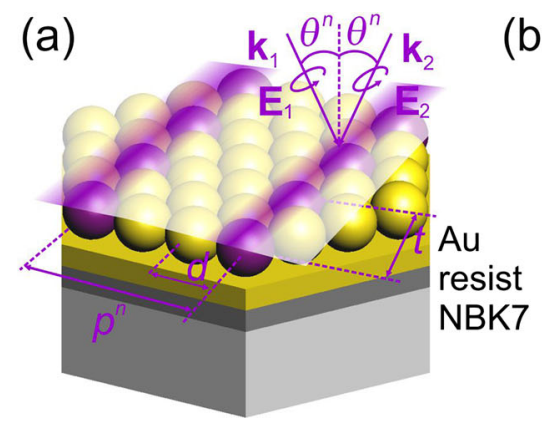

Fig. 1 a Schematic drawing of a gold-resist bilayer coated target that can be structured in IICSM methodology followed by a lift-off procedure: 3D scheme of $p^{n}$ pattern period variation by $\theta^{n}$ polar angle tuning. b Orientation of incidence plane $(\alpha)$ and interference pattern $(\beta)$ to tune gold colloid sphere $d$ diameter-scaled $t$ distance of

\section{Spectral and Near-field Study of Different Patterns}

Similarly, FEM (COMSOL) was used to demonstrate the spectral engineering capabilities of convex patterns that can be fabricated by applying circularly polarized light in IICSM. Complete spectral study of these complex patterns was realized, namely the optical responses of patterns composed of convex gold nano-objects with $45 \mathrm{~nm}$ height were determined (Fig. 2). IICSM can be simplified to generate hexagonal patterns similar to those presented in the previous literature [25, 40-42, 45, 46]. The inspected realistic hexagonal patterns are as follows: hexagonal pattern of (i) nanorings (Fig. 3a, b and d), (ii) horizontal nanocrescents (c)

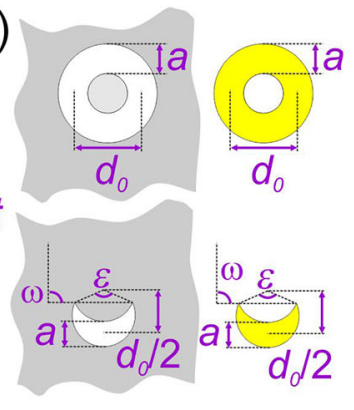

nano-objects. c Characteristic size parameters of the resist pattern and of the transferred gold pattern, the generated features are qualified by the size parameters of $d_{0}$ diameter and $a$ thickness of nanoring and nanocrescent, $\varepsilon$ gap angle and $\omega$ orientation of nanocrescent.

(Fig. 3a, c, e), vertical nanocrescents that are presented in a Supplementary Material). The studied artificial rectangular patterns are as follows: $300 \mathrm{~nm}$ rectangular pattern of (iii) a singlet nanoring (Fig. 4), (iv) a singlet horizontal nanocrescent (Fig. 5), (v) a quadrumer of slightly rotated nanocrescents (Fig. 6). Finally, two different complex rectangular patterns that can be fabricated by utilizing the capabilities of IICSM were analyzed: a (vi) 300-nm (Fig. 7) and a (vii) 600-nm periodic (Fig. 8) rectangular pattern of the same miniarray composed of a central nanoring and a quadrumer of slightly rotated nanocrescents.

To analyze the spectral and near-field properties, the convex patterns were re-illuminated by p-polarized light by

Fig. 2 Normalized $\bar{E}$-field on the upper surface of the gold-resist bilayer coated target to be structured under $\mathrm{Au}$ colloid sphere monolayers illuminated by a single circularly polarized $\lambda=400 \mathrm{~nm}$ beam incident a perpendicularly and $\mathbf{b}$ obliquely, and $\mathbf{c}, \mathbf{d}$ by applying IICSM configuration. The insets show the unit cells of the resulted convex patterns transferred into the gold layer: hexagonal pattern of a nanorings and $\mathbf{b}$ nanocrescents, rectangular pattern of ring-crescent miniarrays with $\mathbf{c} p^{6}=300 \mathrm{~nm}$ $(n=6)$ and $\mathbf{d} p^{12}=600 \mathrm{~nm}$ $(n=12)$ periodicity, by using a two-beam interference pattern that is rotated by $30^{\circ}$ with respect to the (100) colloid sphere monolayer lattice direction in IICSM realization in both cases

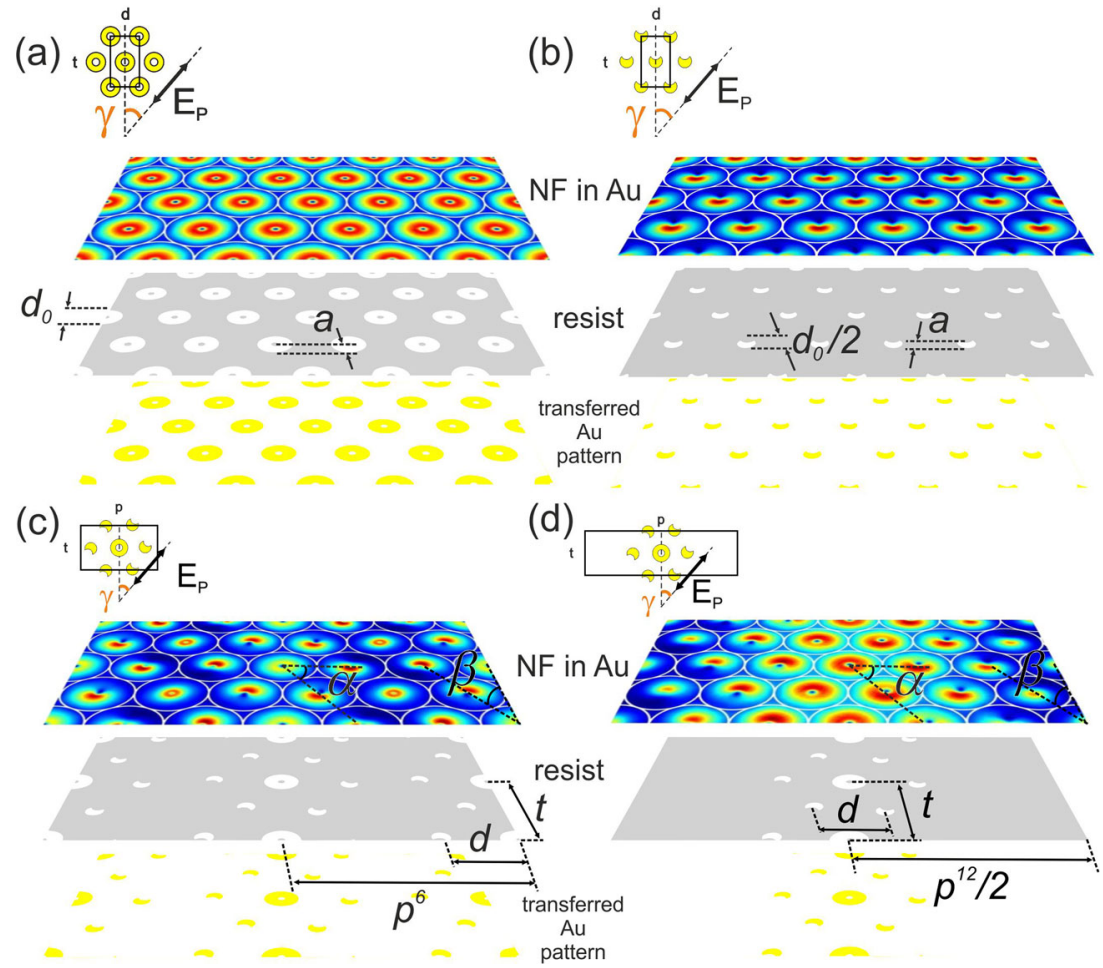


varying the $\gamma$ azimuthal angle, which qualifies the incidence plane orientation with respect to the y axes. In this spectral study, p-polarized light illuminated the convex gold nano-objects in a symmetric environment, supposing that the nanorings and nanocrescents are surrounded by and the nanorings' inner holes are filled with NBK7 glasslike medium (Schott). The wavelength-dependent optical properties of this type of glass are similar to those of typical photoresists, so the same medium was used to ensure a completely symmetric environment. The hexagonal pattern of convex nanorings and nanocrescents has been inspected in $0^{\circ}$ and $90^{\circ}$ azimuthal orientations in order to uncover the characteristic LSPRs supported by the nanoobjects. These $\gamma$ azimuthal angles ensure $\bar{E}$-field oscillation direction along $\left(0^{\circ}\left(90^{\circ}\right)\right)$ and perpendicularly $\left(90^{\circ}\left(0^{\circ}\right)\right)$ to the horizontal (vertical) nanocrescent symmetry axes, that orientations promote $\mathrm{U}$ and $\mathrm{C}$ type LSPR on crescentshaped convex nano-objects in accordance with the literature, respectively $[23,45]$. Both the $0^{\circ} / 16^{\circ}$ and $90^{\circ} / 106^{\circ}$ azimuthal orientations of the rectangular patterns have been inspected, since these promote LSPR as well as surface lattice resonance effects in case of singlet horizontal nanocrescents/LSPR in cases of the quadrumer and either miniarrays. The spectra were computed throughout the 400-1000-nm interval with a $10-\mathrm{nm}$ resolution, at $\varphi=0^{\circ}$ polar angle corresponding to a perpendicular incidence (Fig. 3-8/a).

FEM (COMSOL) was used to inspect the dispersion characteristics of all convex patterns by selecting fractions on the high symmetry path throughout their irreducible Brillouin zone according to those azimuthal orientations that promote uncovering of the LSPR on constituent circular nano-objects and mapping of the photonic modes scattering as well. Accordingly, the dispersion characteristics have been analyzed in $0^{\circ}$ and $90^{\circ}$ azimuthal orientations. In case of dispersion diagram computations, the spectral range was extended similarly through $1000 \mathrm{~nm}$ applying the same $10-\mathrm{nm}$ wavelength resolution as in case of perpendicular incidence, whereas the $\varphi$ incidence angle was also modified from $0^{\circ}$ to $85^{\circ}$ with $5^{\circ}$ steps (Fig. 3d, e and Fig. 4$8 / \mathrm{c}$ ). Wherever needed to uncover all underlying modes on the dispersion graphs, higher resolution complementary calculations were realized with smaller steps.

According to the literature, in case of plasmonic patterns the absorptance spectra are the most informative to find resonances; therefore, the absorptance spectra and the dispersion characteristics computed in p-polarized absorptance are analyzed throughout this paper [15, 59].

During inspection of the near-field phenomena, first, the characteristic charge distribution corresponding to $\mathrm{C}$ and $\mathrm{U}$ resonance has been determined based on previous results in the literature, then the accompanying EM-field has been inspected on the time-averaged $E_{z}$ distribution [26]. The latter promoted to determine the complementary EM-field and charge distributions on concave patterns (Fig. 3b, c and Fig. 4-8/b) [52, 55, 56].

Localized plasmon resonances on the nanorings and nanocrescents are distinguished by using " $\mathrm{r}$ " and " $\mathrm{c}$ " in the abbreviations.

FEM (COMSOL) was used to inspect the application possibilities of the artificial constituent patterns, namely the rectangular patterns of a singlet nanoring and a quadrumer of nanocrescents, as well as of the rectangular pattern of their miniarray to enhance the fluorescence (Fig. 9).

\section{Results and Discussion}

\section{Patterns Achievable in Different Illumination Configurations}

Via illumination of a monolayer composed of 100-nm Au colloid spheres by a homogeneous perpendicularly and obliquely $\left(\theta^{6}=41.8^{\circ}\right)$ incident circularly polarized $\lambda=400$ $\mathrm{nm}$ beam, hexagonal array of uniform nanorings (Fig. 2a) and nanocrescents (Fig. 2b) can be fabricated, respectively. Via IICSM methodology rectangular patterns composed of analogue miniarrays consisted of a central nanoring and satellite nanocrescents with $p^{6}=300 \mathrm{~nm}$ and $p^{12}=600 \mathrm{~nm}$ periodicity can be generated by two interfering circularly polarized beams incident at $\theta^{6}=41.8^{\circ}$ and $\theta^{12}=19.5^{\circ}$ angles, corresponding to $n=6$ and $n=12$ cases (Fig. 2c, d) [49-51].

In our present study, the size parameters of the inspected ring- and crescent-shaped nano-objects, the $\varepsilon$ opening angle, and the $\omega$ orientation of the nanocrescents, as well as the pattern periods, are equal to those of the complementary concave patterns described in our corresponding papers, in order to ensure comparability $[55,56]$. Namely, uniform nanorings with 10-nm and 46-nm (50-nm) inner and outer diameters were inserted into the hexagonal pattern (artificial rectangular pattern of singlet nanorings and both rectangular patterns of analogue miniarrays). The nanocrescents were approximated as the intersections of two cylindrical objects with $25-\mathrm{nm}$ and $20-\mathrm{nm}$ diameter, located at $12.5-\mathrm{nm}$ center distance.

\section{Spectral and Near-field Effects of Different Patterns}

\section{Hexagonal Pattern of Convex Nanorings}

In the case of a hexagonal pattern composed of nanorings, no orientation dependence is expected based on the spherical symmetry of nanorings and on the symmetry properties of hexagonal lattices. As a result, the optical response is 
azimuthal orientation independent; accordingly, the absorptance spectra completely overlap in the two inspected mutually perpendicular azimuthal orientations, namely at $0^{\circ}$ and $90^{\circ}$ azimuthal angles.

On the absorptance of the hexagonal pattern composed of convex nanorings, only a shoulder appears in the interval of particle plasmon resonance (r-PPR, $530 \mathrm{~nm})$ in either of these inspected azimuthal orientations (Fig. 3a). Not only parallel, but also reversed dipoles develop on the inner and outer rim in a noticeable fraction within one cycle of the time-dependent charge distribution, which correlates with the allowed different charge separations that accompany the PPR phenomenon on tiny gold nano-objects. The $E_{z}$ field component is relatively weaker on the outer rim of nanorings in the spectral interval corresponding to r-PPR. After the shoulder, a global maximum appears at $590 \mathrm{~nm}$ both in $90^{\circ}$ and $0^{\circ}$ azimuthal orientations, where exclusively parallel dipoles develop on the inner and outer rim of the ring along the $\bar{E}$-field direction. These are nominated as $\mathrm{r}-\mathrm{C}$ and $\mathrm{r}-\mathrm{U}$ resonance in order to ensure comparability with the resonances on nanocrescents. The $E_{z}$ distribution indicates more commensurate lobes on the inner and outer rims parallel to the $\bar{E}$-field direction, both in $0^{\circ}$ and $90^{\circ}$ azimuthal orientations (Fig. 3b).

\section{Hexagonal Pattern of Horizontal Convex Nanocrescents}

\section{Hexagonal Pattern of Horizontal Convex Nanocrescents in C Orientation}

On the absorptance of the hexagonal pattern composed of horizontal convex nanocrescents, a local maximum (550 $\mathrm{nm})$, a shoulder $(590 \mathrm{~nm})$, and a global maximum $(650$ nm) appears in $90^{\circ}$ azimuthal orientation, where the $\bar{E}$ field direction is perpendicular to the symmetry axis of the (a)
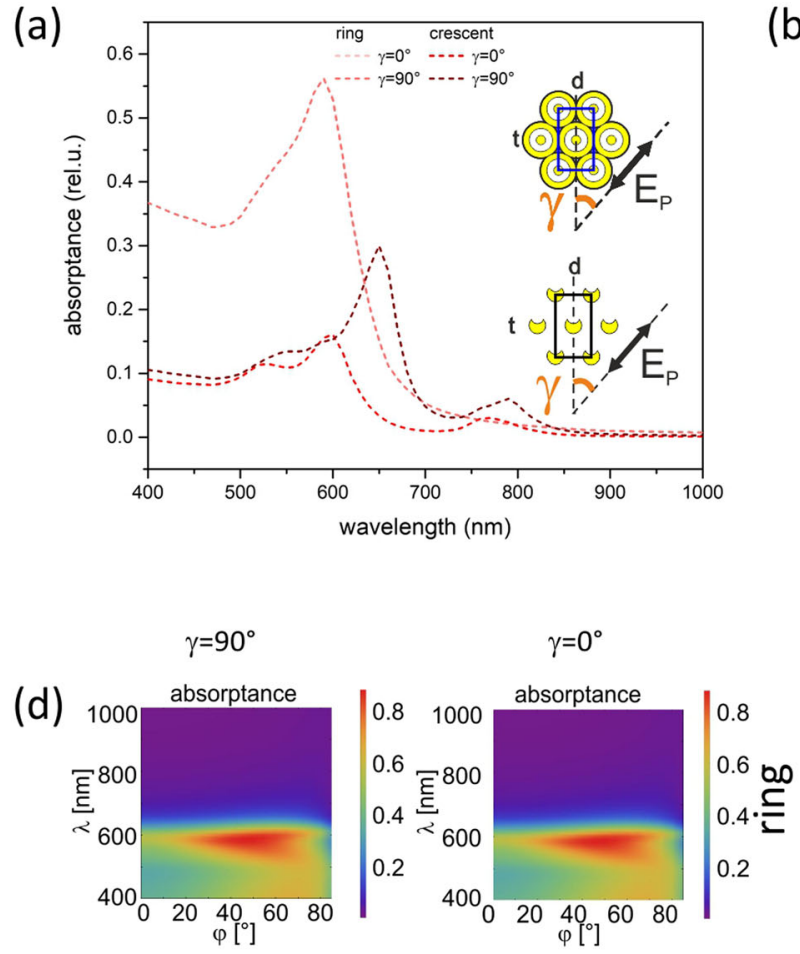

(e)

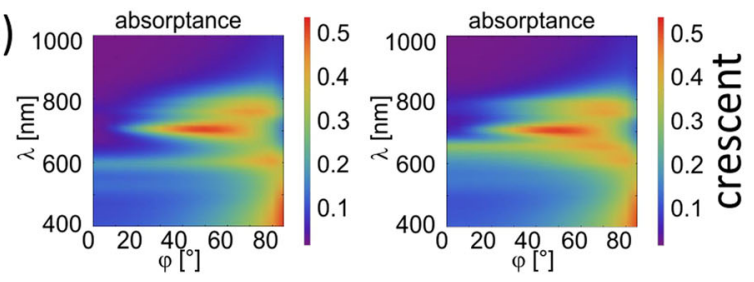

Fig. 3 Hexagonal patterns composed of convex nanorings and horizontal nanocrescents: a absorptance spectra, $E_{z}$ field component and charge distribution in (top) $90^{\circ}$ and (bottom) $0^{\circ}$ azimuthal orientation of $\mathbf{b}$ nanorings and $\mathbf{c}$ nanocrescents, dispersion characteristics
$\mathrm{Ez}$

(b)

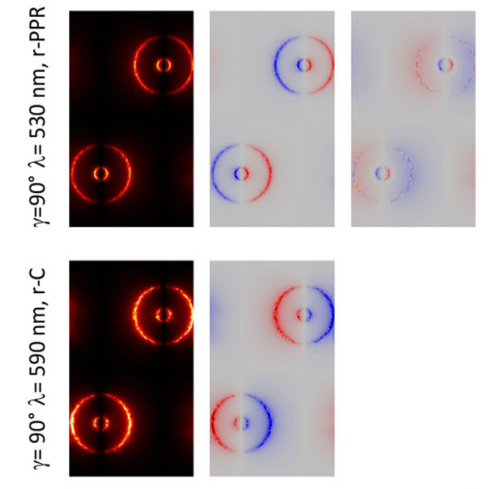

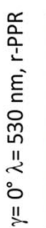
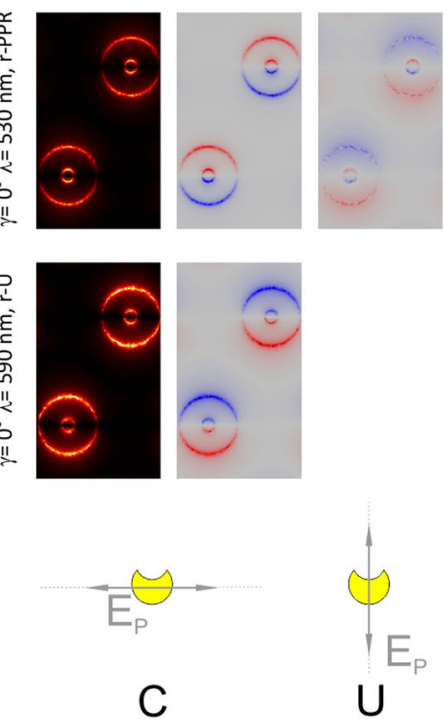

(c)

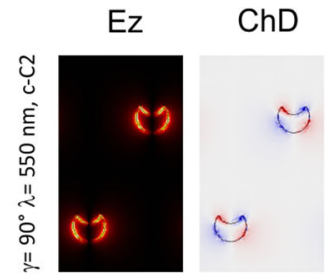

นิ
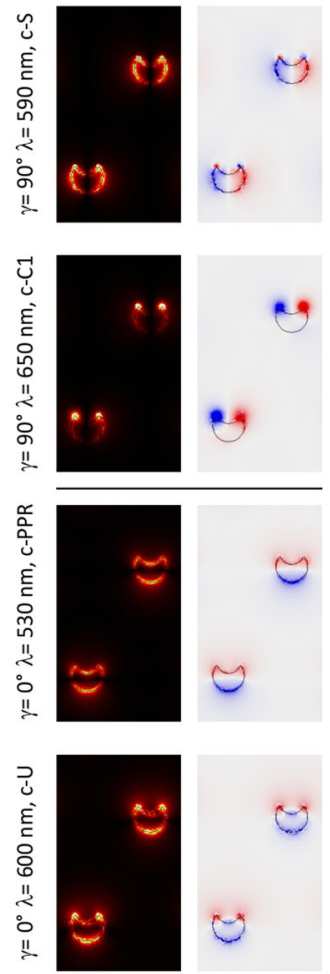

computed in (left) $90^{\circ}$ and (right) $0^{\circ}$ azimuthal orientation of $\mathbf{d}$ nanorings and e nanocrescents. Insets: schematic drawings of the unit cells and $\mathrm{C}$ and $\mathrm{U}$ illumination configurations 
nanocrescents; therefore, it is nominated as $\mathrm{C}$ orientation in accordance with the literature [23, 45] (Fig. 3a). The charge distribution is quadrupolar at the local maximum originating from the spectrally overlapping and interacting c-PPR and c-C2 resonance. Accordingly, there are four lobes on the $E_{z}$ distribution. At the shoulder, a hexapolar charge distribution is observable, which is accompanied by stronger charge accumulation at the tips. As a result, four weak and two intense lobes are distinguishable with intensity maxima on the nanocrescent tips on the $E_{z}$ distribution. At the global maximum, dipoles arise along the $\bar{E}$-field direction, which unambiguously reveals the c$\mathrm{C} 1$ resonance. Exclusively, the two tips of the nanocrescents are overlap with intense areas on the $E_{z}$ distribution at this maximum (Fig. 3c, top). Both the charge and the $E_{z}$ distribution indicate that the shoulder originates from the interaction of the quadrupolar and dipolar modes excitable at the neighboring extrema. Namely, the four weaker and two stronger lobes inherited from c-C2 and c-C1 resonance create two separated composite lobes with their centers at the nanocrescent tips. The small local maximum after the global maximum originates from a resonance that can be excited azimuthal orientation dependently on the tips of the nanocrescents. Both the strength and the exact location of these extrema strongly depend on the mesh density; therefore, a mesh quality ensuring separation from the well-defined LSPRs has been selected. Further details are described in the Supplementary Material.

\section{Hexagonal Pattern of Horizontal Convex Nanocrescents in $\mathrm{U}$ Orientation}

In comparison, on the absorptance of the hexagonal pattern composed of horizontal convex nanocrescents, a local maximum $(530 \mathrm{~nm})$ is followed by the global maximum $(600 \mathrm{~nm})$ in $0^{\circ}$ azimuthal orientation, where the $\bar{E}$-field direction is along the symmetry axis of the nanocrescents; therefore, it is nominated as $U$ orientation in accordance with the literature [23, 45] (Fig. 3a). Dipolar charge distribution is observable already at the local maximum corresponding to the particle plasmon resonance (c-PPR). Accordingly, there are two lobes on the $E_{z}$ distribution. At the global maximum dipoles arise along the $\bar{E}$-field direction with stronger charge accumulation on the tips, which unambiguously reveals the $\mathrm{c}-\mathrm{U}$ resonance (Fig. 3c, bottom).

The charge distribution is rearranged with respect to the c-PPR in such a way that the two tips and the long arch of the nanocrescents are the overlap with the most intense areas on the $E_{z}$ distribution at the global maximum. One has to emphasize that at both maxima the charge distribution is monopolar (dipolar) in 60\% (40\%) fraction of one cycle of the time-dependent charge distribution (Fig. 3c, bottom).

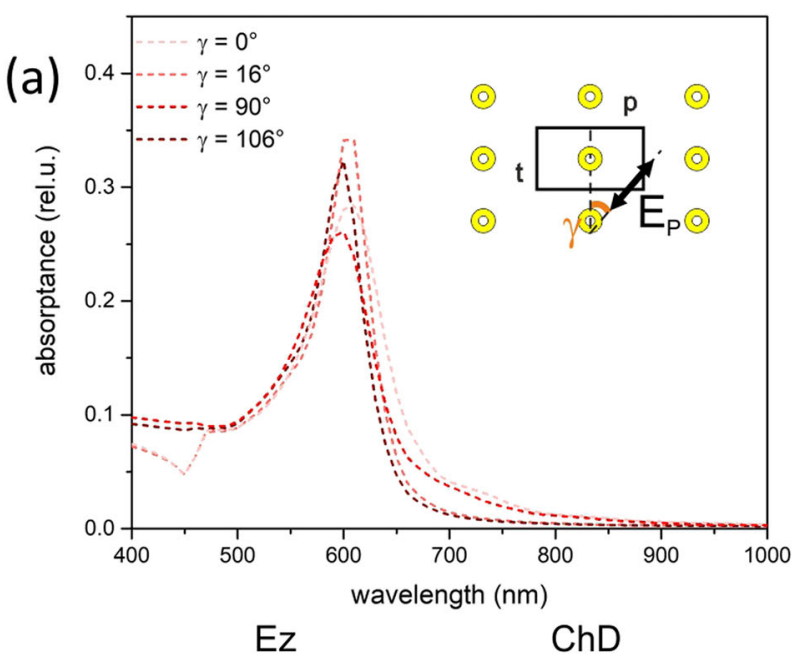

(b)

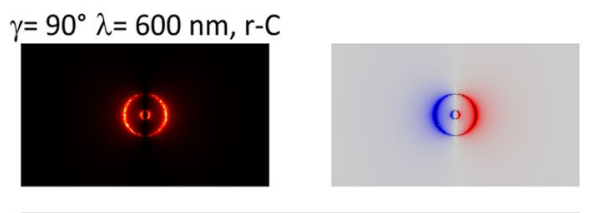

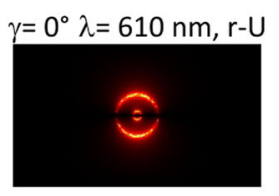

$\gamma=90^{\circ}$
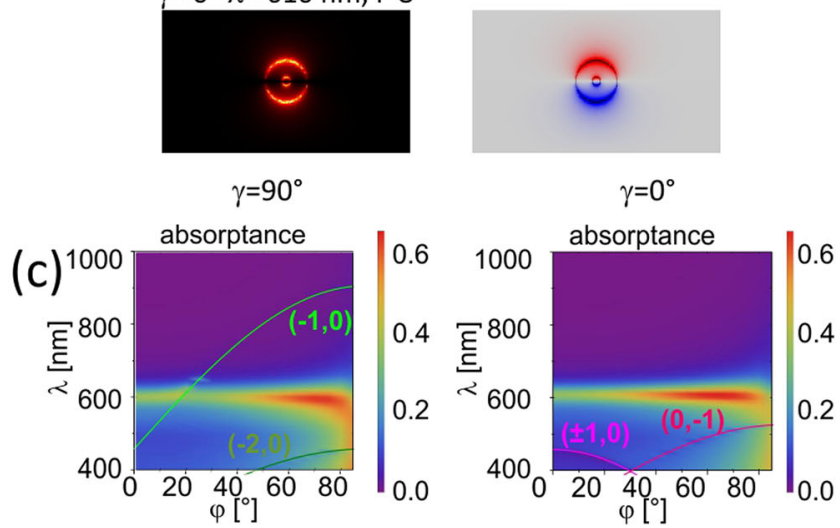

Fig. 4 Rectangular $p=300 \mathrm{~nm}$ periodic pattern composed of singlet convex nanorings: a absorptance spectra, $\mathbf{b} E_{z}$ field component and charge (ChD) distribution in (top) $90^{\circ}$ and (bottom) $0^{\circ}$ azimuthal orientation (note that ESM is available in (top) $106^{\circ}$ and (bottom) $16^{\circ}$ azimuthal orientation; c dispersion characteristics computed in (left) $90^{\circ}$ and (right) $0^{\circ}$ azimuthal orientation. Inset: schematic drawing of the unit cell

\section{Rectangular 300-nm Periodic Pattern of a Singlet Convex Nanoring}

\section{Rectangular 300-nm Periodic Pattern of a Singlet Convex Nanoring in C Orientation of Nanocrescents}

When a singlet convex nanoring of similar geometry as those inspected in the hexagonal pattern is arranged into a 300-nm rectangular pattern, on the absorptance no extremum/a tiny shoulder $(-/ 530 \mathrm{~nm})$ and a global maximum $(600 \mathrm{~nm} / 600 \mathrm{~nm})$ appears in $90^{\circ} / 106^{\circ}$ azimuthal orientation, which is the $\mathrm{C}$ orientation of horizontal singlet/slightly rotated quadrumer nanocrescents (Fig. 4a). 
The charge distribution is dipolar at the tiny shoulder corresponding to the particle plasmon resonance (r-PPR, not shown). At the global maximum, parallel dipoles develop on the inner and outer rim and the $E_{z}$ lobes are aligned along the $\bar{E}$-field direction. This peak originates from the r$\mathrm{C}$ resonance on the convex nanoring, which is expected to be insensitive to the $\bar{E}$-field direction based on the spherical symmetry of the convex singlet nanoring (Fig. 4b, top).

\section{Rectangular 300-nm Periodic Pattern of a Singlet Convex Nanoring in U Orientation of Nanocrescents}

In comparison, on the absorptance of the $300-\mathrm{nm}$ periodic rectangular pattern composed of singlet convex nanorings, no extremum/a tiny shoulder $(-/ 530 \mathrm{~nm})$ and a global maximum $(610 \mathrm{~nm} / 610 \mathrm{~nm})$ appear in $0^{\circ} / 16^{\circ}$ azimuthal orientation, which is the $\mathrm{U}$ orientation of horizontal singlet/slightly rotated quadrumer nanocrescents (Fig. 4a). The charge distribution is dipolar at the tiny shoulder corresponding to the particle plasmon resonance (r-PPR, not shown). At the global maximum, parallel dipoles arise on the inner and outer rim and the $E_{z}$ field component enhancement maxima are aligned along the $\bar{E}$-field direction, which reveal the r-U resonance on the convex singlet nanoring (Fig. 4b, bottom).

\section{Rectangular 300-nm Periodic Pattern of a Horizontal Singlet Convex Nanocrescent}

\section{Rectangular 300-nm Periodic Pattern of a Horizontal Singlet Convex Nanocrescent in C Orientation}

When a horizontal singlet convex nanocrescent of the same shape as that inspected in hexagonal pattern is arranged into a 300-nm rectangular pattern, on the absorptance a local maximum/modulation $(550 \mathrm{~nm} / 550 \mathrm{~nm})$ is followed by a shoulder/local maximum $(620 \mathrm{~nm} / 630 \mathrm{~nm})$ and a global maximum $(660 \mathrm{~nm} / 670 \mathrm{~nm})$ appears in $\mathrm{C}$ orientation $\left(90^{\circ}\right) /$ close to it $\left(106^{\circ}\right)$ (Fig. 5a).

The charge distribution is quadrupolar at the local maximum appearing slightly above the particle plasmon resonance, which is the $\mathrm{c}-\mathrm{C} 2$ resonance on the horizontal singlet convex nanocrescent in $90^{\circ}$ azimuthal orientation. Accordingly, there are four lobes on the $E_{z}$ field distribution. At the shoulder/local maximum, the charge distribution is still hexapolar/quadrupolar in $90^{\circ} / 106^{\circ}$ azimuthal orientation; however, the charge accumulation is stronger at the tips. Accordingly, two intense and four/two weak $E_{z}$ lobes are observable, with intensity maxima at the tips. At the global maximum, dipoles arise on the nanocrescent tips along the $\bar{E}$-field direction, which reveals the pure c-C1 resonance on the horizontal singlet convex nanocrescent in $90^{\circ}$ azimuthal orientation. The $E_{z}$ field component indicates two separated lobes on the nanocrescent tips (Fig. 5b, top).
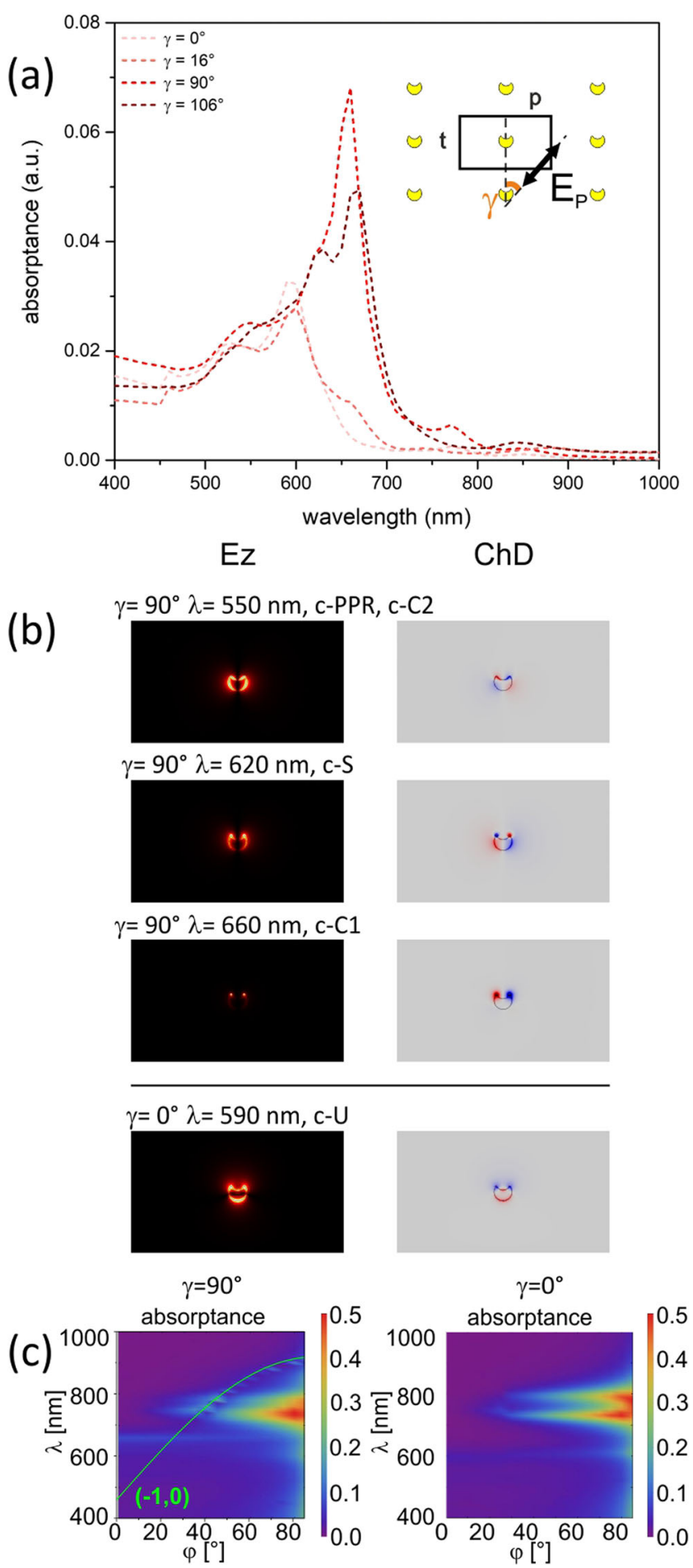

Fig. 5 Rectangular $p=300 \mathrm{~nm}$ periodic pattern composed of horizontal singlet convex nanocrescents: a absorptance spectra, b $E_{z}$ field component and charge (ChD) distribution in (top) $90^{\circ}$ and (bottom) $0^{\circ}$ azimuthal orientation (note that ESM is available in (top) $106^{\circ}$ and (bottom) $16^{\circ}$ azimuthal orientation; $\mathbf{c}$ dispersion characteristics computed in (left) $90^{\circ}$ and (right) $0^{\circ}$ azimuthal orientation. Inset: schematic drawing of the unit cell 
The shoulder in $\mathrm{C}$ orientation originates from the interaction of the c-C 2 and c-C1 modes at $90^{\circ}$ azimuthal angle. Due to the $\bar{E}$-field component along the symmetry axis close to $\mathrm{C}$ orientation, the c-U resonance is cross-coupled; as a result, a maximum rather than a shoulder appears in $106^{\circ}$ azimuthal orientation in the spectral interval, which overlaps with the c-U band (Fig. $5 \mathrm{a}$ and $5 \mathrm{~b}$, top).

\section{Rectangular 300-nm Periodic Pattern of a Horizontal Singlet Convex Nanocrescent in U Orientation}

In comparison, on the absorptance of the rectangular pattern composed of a horizontal singlet convex nanocrescent, a local maximum appearing at the particle plasmon resonance $(530 \mathrm{~nm} / 540 \mathrm{~nm})$ is followed by a global maximum (590 $\mathrm{nm} / 600 \mathrm{~nm})$ in $\mathrm{U}$ orientation $\left(0^{\circ}\right) /$ close to it $\left(16^{\circ}\right)$; in addition to this, a shoulder appears at $660 \mathrm{~nm}$ in $16^{\circ}$ azimuthal orientation (Fig. 5a). The charge distribution is dipolar already at the local maximum appearing at the particle plasmon resonance (c-PPR, not shown). At the global maximum, dipoles arise on the nanocrescent tips along the $\bar{E}$-field direction, which reveals the c-U resonance on the convex singlet nanocrescent in $0^{\circ}$ azimuthal orientation. The $E_{z}$ field component indicates two lobes, one localized on the tips and the other on the larger arch of nanocrescents. Caused by the rearrangement of the charge distribution with respect to cPPR, the $E_{z}$ field component is less intense on the smaller arch of the nanocrescent, similarly to the hexagonal pattern. At the shoulder that follows the global maximum in $16^{\circ}$ azimuthal orientation asymmetrical dipolar charge distribution develops; moreover, the charge becomes different on the two tips. The shoulder is resulted from the appearance of the $\bar{E}$-field component in $16^{\circ}$ azimuthal orientation along the tips, which cross-couples the c-C1 resonance. This is accompanied by two separated $E_{z}$ field lobes, one is asymmetrically arranged on the tips, whereas the other on the larger arch of the nanocrescent (Fig. 5b, bottom).

\section{Rectangular 300-nm Periodic Pattern of a Quadrumer of Convex Nanocrescents}

\section{Rectangular 300-nm Periodic Pattern of a Quadrumer of Convex Nanocrescents in C Orientation}

When quadrumers composed four slightly rotated convex nanocrescents are arranged into a 300-nm rectangular pattern, on their absorptance a local maximum $(550 \mathrm{~nm} / 550$ $\mathrm{nm})$ is followed by a shoulder $(590 \mathrm{~nm} / 600 \mathrm{~nm})$ and a global maximum $(650 \mathrm{~nm} / 650 \mathrm{~nm})$ appears close to $\left(90^{\circ}\right)$ /in $\mathrm{C}$ orientation $\left(106^{\circ}\right)$ (Fig. 6a). Compared to the rectangular pattern of a singlet nanocrescent, all extrema except the first are blue shifted caused by their interaction. The charge distribution is quadrupolar at the local maximum appearing slightly above the particle plasmon resonance, which reveals the $\mathrm{c}-\mathrm{C} 2$ resonance on the nanocrescents

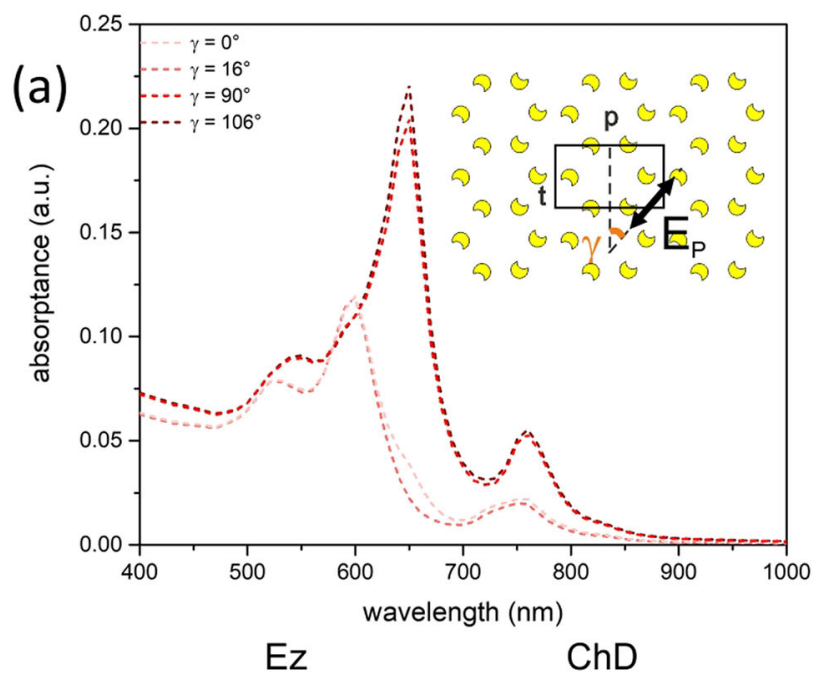

(b)
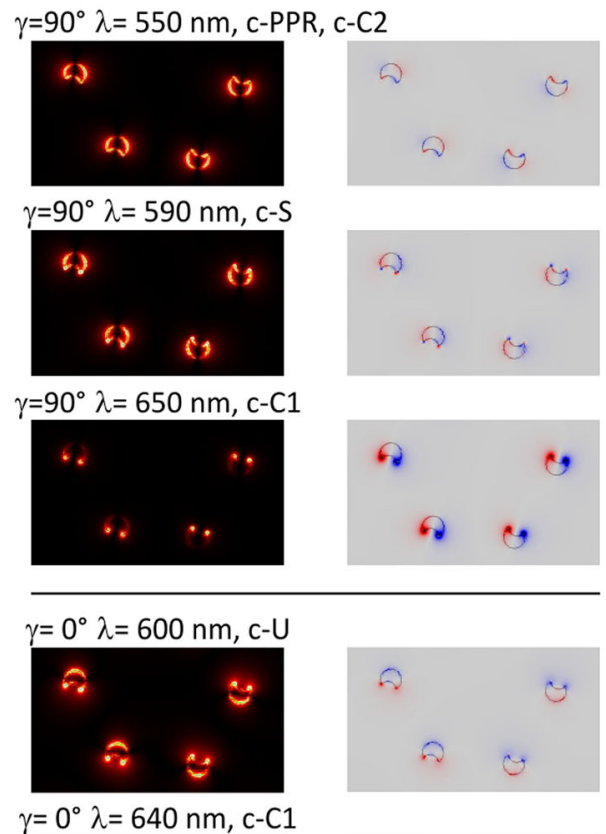

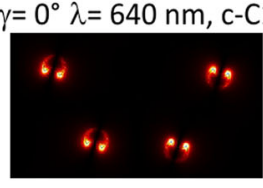

$\gamma=90^{\circ}$
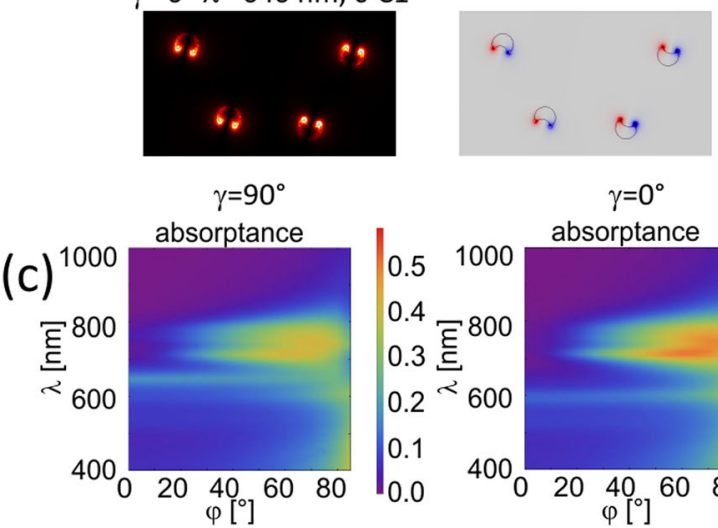

$\gamma=0^{\circ}$

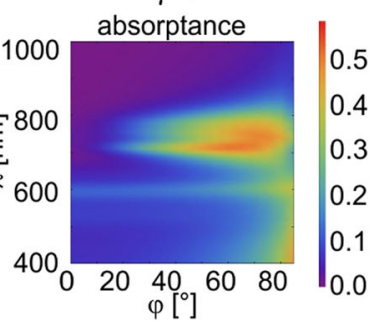

Fig. 6 Rectangular $p=300 \mathrm{~nm}$ periodic pattern composed of quadrumer convex nanocrescents: a absorptance spectra, b $E_{z}$ and charge $(\mathrm{ChD})$ distribution in (top) $90^{\circ}$ and (bottom) $0^{\circ}$ azimuthal orientation (note that ESM is available in (top) $106^{\circ}$ and (bottom) $16^{\circ}$ azimuthal orientation; c dispersion characteristics computed in (left) $90^{\circ}$ and (right) $0^{\circ}$ azimuthal orientation. Inset: schematic drawing of the unit cell 
quadrumer in $106^{\circ}$ azimuthal orientation. Accordingly, there are four lobes on the $E_{z}$ distribution. At the shoulder, the charge distribution exhibits a hexapolar modulation/it is completely hexapolar in $90^{\circ} / 106^{\circ}$ azimuthal orientation. Despite the $\bar{E}$-field component appearance along the symmetry axis of slightly rotated nanocrescents close to C orientation $\left(90^{\circ}\right)$, the c-U resonance is not efficiently crosscoupled, only a preference to quadrupolar charge separation is noticeable. The charge accumulation is stronger at the nanocrescent tips. Accordingly, two intense and four weak lobes appear on the $E_{z}$ distribution, with intensity maxima at the nanocrescent tips. At the global maximum, dipoles arise on the nanocrescent tips along the $\bar{E}$-field direction, which reveals the pure c-C1 resonance on the convex nanocrescents' quadrumer in $106^{\circ}$ azimuthal orientation. The $E_{z}$ field component indicates two separated lobes on the nanocrescent tips. Both the charge and the $E_{z}$ field distributions prove that the shoulder originates from the interaction of the c-C2 and c-C1 modes (Fig. 6b, top).

\section{Rectangular 300-nm Periodic Pattern of a Quadrumer of Convex Nanocrescents in U Orientation}

In comparison, on the absorptance of the rectangular pattern composed of four convex nanocrescents, a local maximum appearing at the particle plasmon resonance (530 $\mathrm{nm} / 530 \mathrm{~nm})$ is followed by a global maximum $(600 \mathrm{~nm} / 600$ $\mathrm{nm})$ close to $\left(0^{\circ}\right) / \mathrm{in} \mathrm{U}$ orientation $\left(16^{\circ}\right)$; in addition to this, a shoulder is observable at $650 \mathrm{~nm}$ in $0^{\circ}$ azimuthal orientation (Fig. 6a). The charge distribution is dipolar already at the local maximum appearing at the particle plasmon resonance (c-PPR, not shown). At the global maximum, dipoles arise on the nanocrescent tips along the $\bar{E}$-field direction, which reveals the c-U resonance on the quadrumer of four convex nanocrescents in $16^{\circ}$ azimuthal orientation. The $E_{z}$ field component indicates two lobes, one asymmetrically/symmetrically arranged on the nanocrescent tips and the other on their larger arch.

The shoulder after the global maximum in $0^{\circ}$ azimuthal orientation originates from the cross-coupling of the cC1 mode due to the $\bar{E}$-field component perpendicularly to the nanocrescent symmetry axis. Accordingly, the charge distribution is dipolar with different charges on the nanocrescent tips, which is accompanied by two separated $E_{z}$ lobes (Fig. 6b, bottom).

\section{Rectangular 300-nm Periodic Pattern of a Complex Convex Miniarray}

\section{Rectangular 300-nm Periodic Pattern of a Complex Convex Miniarray in C Orientation}

When a $300-\mathrm{nm}$ periodic rectangular pattern is composed of a convex miniarray consisted of a central nanoring and a quadrumer of nanocrescents, on their absorptance a

shoulder $(550 \mathrm{~nm} / 550 \mathrm{~nm})$ is followed by a global $(600$ $\mathrm{nm} / 600 \mathrm{~nm})$ and a local maximum $(650 \mathrm{~nm} / 650 \mathrm{~nm})$ close to $\left(90^{\circ}\right)$ /in $\mathrm{C}$ orientation $\left(106^{\circ}\right)$ (Fig. $\left.7 \mathrm{a}\right)$. At the shoulder, the charge distribution consists of reversed dipoles on

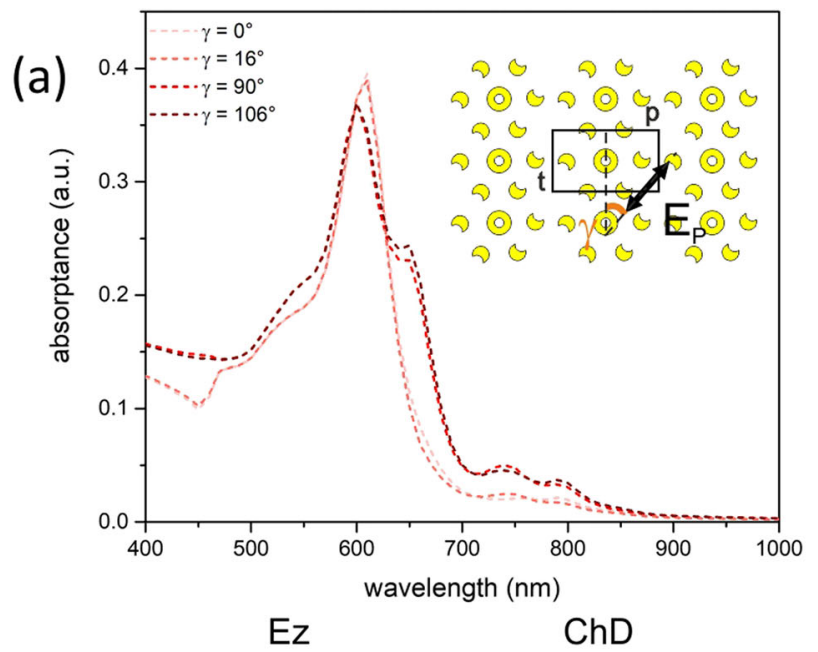

(b)

$\gamma=90^{\circ} \lambda=550 \mathrm{~nm}, \mathrm{r} \& \mathrm{c}-\mathrm{PPR}, \mathrm{c}-\mathrm{C} 2$

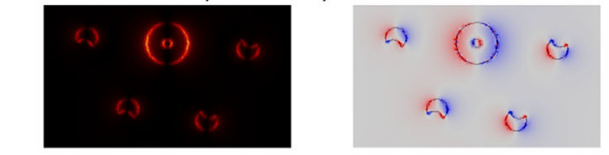

$\gamma=90^{\circ} \lambda=600 \mathrm{~nm}, \mathrm{r}-\mathrm{C}, \mathrm{c}-\mathrm{S}$

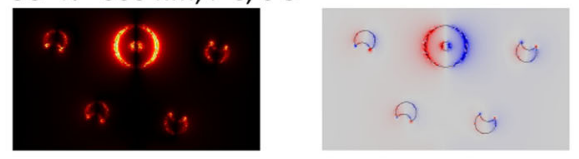

$\gamma=90^{\circ} \lambda=650 \mathrm{~nm}, \mathrm{c}-\mathrm{C} 1$

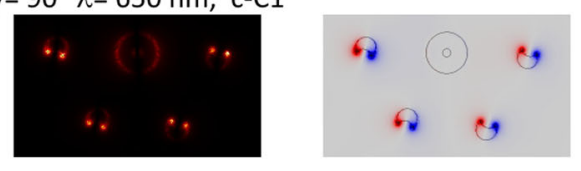

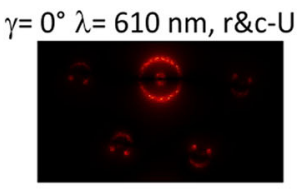

$\gamma=90^{\circ}$
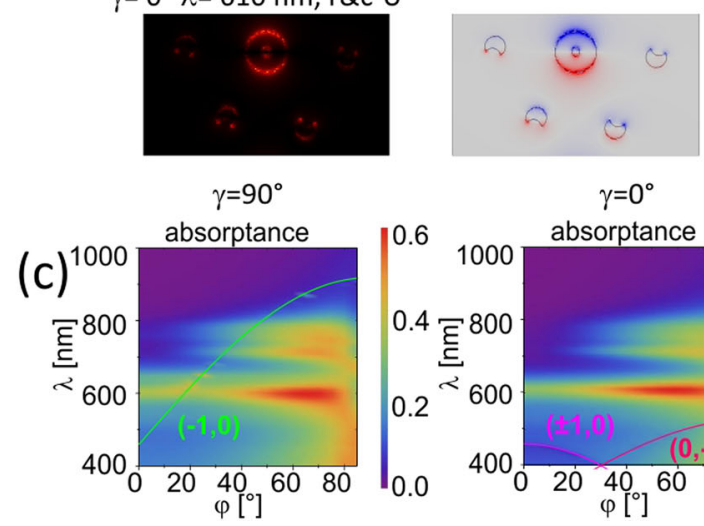

$\gamma=0^{\circ}$

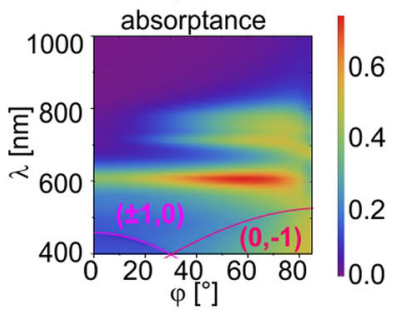

Fig. 7 Rectangular $p=300 \mathrm{~nm}$ periodic pattern composed of a complex convex miniarray: a absorptance spectra, $\mathbf{b} E_{z}$ field component and charge (ChD) distribution in (top) $90^{\circ}$ and (bottom) $0^{\circ}$ azimuthal orientation (note that ESM is available in (top) $106^{\circ}$ and (bottom) $16^{\circ}$ azimuthal orientation; $\mathbf{c}$ dispersion characteristics computed in (left) $90^{\circ}$ and (right) $0^{\circ}$ azimuthal orientation. Inset: schematic drawing of the unit cell 
the nanoring (r-PPR), whereas it is quadrupolar on the nanocrescents, which reveals the $\mathrm{c}-\mathrm{C} 2$ resonance on the nanocrescents. Accordingly, there are commensurate lobes on the inner and outer rim of the nanoring, and four lobes on the nanocrescents on the $E_{z}$ distribution.

At the global maximum, strong parallel dipoles appear on the inner and outer rim of the nanoring, whereas the charge distribution exhibits a slightly/unambiguously hexapolar modulation on the nanocrescents, which is accompanied by stronger charge accumulation at the tips. According to the $\mathrm{r}-\mathrm{C}$ ring-resonance origin of this maximum, relatively stronger $E_{z}$ lobes appear especially on the outer rim of the nanoring, whereas two intense and four weak $E_{z}$ lobes are observable on the nanocrescents, which exhibit intensity maxima on the tips that are relatively weaker compared to those on the nanoring. At the local maximum, no significant charge separation occurs on the nanoring, whereas strong dipoles arise on the nanocrescent tips along the $\bar{E}$-field direction, which reveals the pure c-C1 resonance on the convex nanocrescent quadrumer in $106^{\circ}$ azimuthal orientation. Accordingly, the $E_{z}$ field component indicates week lobes on the outer rim of the ring, and two separated strong lobes on the nanocrescent tips (Fig. 7b, top).

Comparison to the rectangular pattern of a singlet nanoring and a quadrumer of four nanocrescents proves that the complex miniarray response originates from the sum of the c-C2 and c-C1 modes on the quadrumer of nanocrescents, and from the r-PPR and $\mathrm{r}-\mathrm{C}$ resonance of the nanoring, the former (latter) overlaps with the c-PPR (mixed mode originating from the interacting c-C2 and c-C1 resonances) on the nanocrescents (Fig. 7b, top).

\section{Rectangular 300-nm Periodic Pattern of a Complex Convex Miniarray in U Orientation}

In comparison, on the absorptance of the 300-nm periodic rectangular pattern composed of a complex convex miniarray, a shoulder appearing at the particle plasmon resonance $(540 \mathrm{~nm} / 540 \mathrm{~nm})$ is followed by a global maximum $(610 \mathrm{~nm} / 610 \mathrm{~nm})$ close to $\left(0^{\circ}\right) /$ in $U$ orientation $\left(16^{\circ}\right)$ (Fig. 7a). There are parallel/reversed dipoles on the inner and outer rim of the nanoring, whereas the charge distribution is quadrupolar/dipolar on the nanocrescents at the shoulder appearing at the PPR (r-PPR and c-PPR, not shown in Fig. 7b, bottom). At the global maximum, parallel dipoles arise on the inner and outer rim of the nanoring, and dipoles arise on the nanocrescents along the $\bar{E}$-field direction, which reveals the $\mathrm{r}-\mathrm{U}$ and $\mathrm{c}-\mathrm{U}$ resonance on the components of the miniarray in $16^{\circ}$ azimuthal orientation.

The $E_{z}$ field component indicates relatively stronger lobes on the outer rim, and two strong lobes appear on the nanocrescents, one asymmetrically/symmetrically on the tips and the other on their larger arch. The miniarray response originates from the sum of the r-PPR and r-U resonance on the nanoring and of the c-PPR and c-U modes on the quadrumer of four nanocrescents, which overlap spectrally in couples (Fig. 7b, bottom).

\section{Rectangular 600-nm Periodic Pattern of a Complex Convex Miniarray}

\section{Rectangular 600-nm Periodic Pattern of a Complex Convex Miniarray in C Orientation}

When two-times larger 600-nm periodic rectangular pattern is composed of a convex miniarray consisted of the central nanoring and quadrumer of nanocrescents, on their absorptance a shoulder $(550 \mathrm{~nm} / 550 \mathrm{~nm})$ is followed by a global maximum $(600 \mathrm{~nm} / 600 \mathrm{~nm})$ and by a shoulder/local maximum $(650 \mathrm{~nm} / 650 \mathrm{~nm})$ close to $\left(90^{\circ}\right) /$ in $\mathrm{C}$ orientation $\left(106^{\circ}\right)$ (Fig. 8a). The charge distribution exhibits reversed dipoles on the nanoring (r-PPR), whereas it is quadrupolar on the nanocrescents at the shoulder, which reveals the c-C2 resonance on the miniarray in $106^{\circ}$ azimuthal orientation. Accordingly, there are commensurate lobes on the inner and outer rim of the nanoring along the $\bar{E}$-field direction, and four lobes on the nanocrescents on the $E_{z}$ distribution. At the global maximum, strong parallel dipoles appear on the inner and outer rim of the nanoring along the $\bar{E}$ field direction, which reveal the $\mathrm{r}-\mathrm{C}$ resonance. In contrast, in the spectral interval of former shoulders, the charge distribution exhibits a slight/unambiguous mixed mode related hexapolar modulation on the nanocrescents, which is accumulated at the tips. According to the ring-origin of this maximum, relatively stronger $E_{z}$ lobes appear especially on the outer rim of the nanoring along the $\bar{E}$-field direction, whereas two intense and four weak $E_{z}$ lobes are observable on the nanocrescents, which exhibit intensity maxima on the tips that are relatively weaker than those on the nanoring. At the shoulder/local maximum negligible charge separation occurs on the nanoring, whereas strong dipoles arise on the nanocrescent tips along the $\bar{E}$-field direction, which reveals the pure $\mathrm{c}-\mathrm{C} 1$ resonance on the convex nanocrescents' quadrumer in the miniarray in $106^{\circ}$ azimuthal orientation. Accordingly, the $E_{z}$ field component indicates significantly weaker lobes on the outer rim of the ring, and two very strong lobes on the nanocrescent tips. Similarly to 300$\mathrm{nm}$ rectangular pattern, on the $600-\mathrm{nm}$ periodic pattern of the analogue miniarray the optical response originates again from the sum of the c-C2 and c-C1 modes on the nanocrescents and from the r-PPR and $\mathrm{r}-\mathrm{C}$ resonance of the nanoring, the former (latter) overlaps with the c-PPR (mixed mode originating from the interacting c-C2 and c-C1 modes) on the quadrumer of nanocrescents (Fig. 8b, top). 
(a)

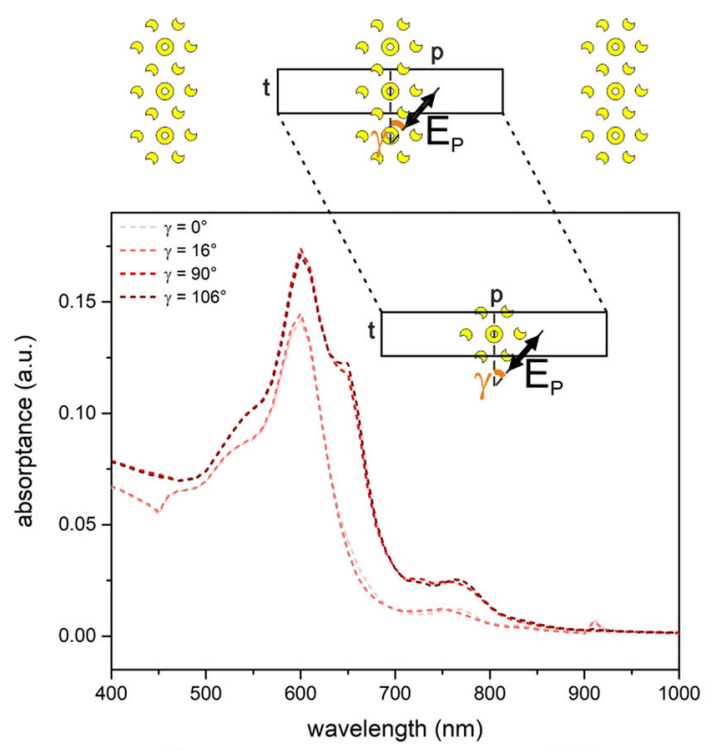

$\mathrm{Ez}$

(b)

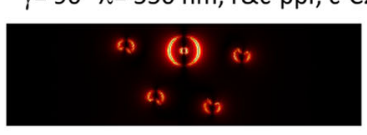

$\gamma=90^{\circ} \lambda=600 \mathrm{~nm}, \mathrm{r}-\mathrm{C}, \mathrm{c}-\mathrm{S}$
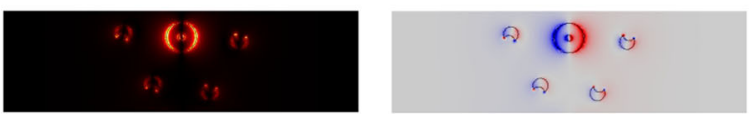

$\gamma=90^{\circ} \lambda=650 \mathrm{~nm}, \mathrm{c}-\mathrm{C} 1$
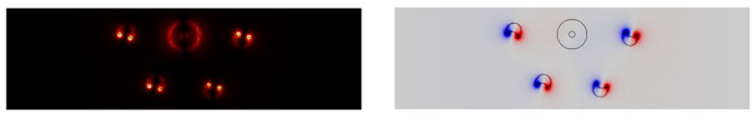

$\gamma=0^{\circ} \lambda=600 \mathrm{~nm}, \mathrm{r} \& \mathrm{c}-\mathrm{U}$
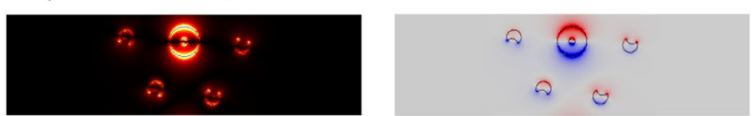

$\gamma=0^{\circ} \lambda=910 \mathrm{~nm}, \mathrm{r} \& \mathrm{c}-\mathrm{RA}$
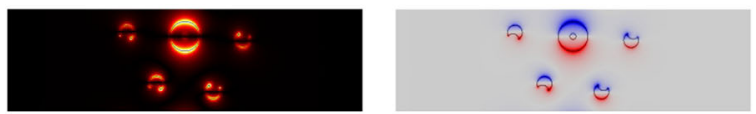

$\gamma=90^{\circ}$

(c)
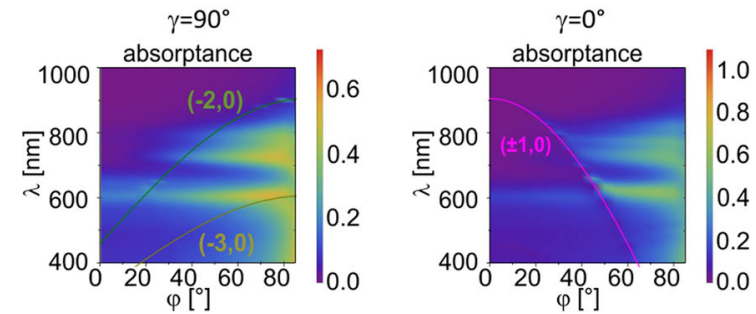

Fig. 8 Rectangular $p^{\prime}=600 \mathrm{~nm}$ periodic pattern composed of a complex convex miniarray: a absorptance spectra, b $E_{z}$ field component and charge (ChD) distribution in (top) $90^{\circ}$ and (bottom) $0^{\circ}$ azimuthal orientation (note that ESM is available in (top) $106^{\circ}$ and (bottom) $16^{\circ}$ azimuthal orientation; $\mathbf{c}$ dispersion characteristics computed in b $90^{\circ}$ and $\mathbf{c} 0^{\circ}$ azimuthal orientation. Inset: schematic drawing of the unit cell

\section{Rectangular 600-nm Periodic Pattern of Complex Convex Miniarray in U Orientation}

In comparison, on the absorptance of the $600-\mathrm{nm}$ periodic rectangular pattern composed of a complex convex miniarray, a shoulder appearing at the particle plasmon resonance $(530 \mathrm{~nm} / 530 \mathrm{~nm})$ is followed by a global maximum $(600 \mathrm{~nm} / 600 \mathrm{~nm})$, moreover a tiny modulation also appears at larger wavelength $(910 \mathrm{~nm} / 910 \mathrm{~nm})$ close to $\left(0^{\circ}\right) /$ in U orientation $\left(16^{\circ}\right)$ (Fig. 8a).

There are parallel/reversed dipoles on the inner and outer rim of the nanoring, whereas the charge distribution is quadrupolar/dipolar on the nanocrescents at the shoulder appearing at the particle plasmon resonance (r-PPR and c-PPR, not shown in Fig. 8b, bottom). At the global maximum, parallel dipoles arise on the inner and outer rim of the nanoring, and dipoles arise on the nanocrescents, all are aligned along the $\bar{E}$-field direction, which reveals the $\mathrm{r}-\mathrm{U}$ and $\mathrm{c}-\mathrm{U}$ resonance on the miniarray in $16^{\circ}$ azimuthal orientation.

The $E_{z}$ field component indicates relatively stronger lobes on the outer rim of the nanoring, and two strong lobes on the nanocrescents, one asymmetrically/symmetrically arranged on the tips and the other one on their larger arch. The miniarray response originates again from the sum of the r-PPR and $\mathrm{r}-\mathrm{U}$ resonance on the nanoring and the c-PPR and c-U modes on the nanocrescents in quadrumer, which overlap spectrally in couples (Fig. 8b, bottom).

At the tiny modulation related to Rayleigh anomaly, i.e., diffractive coupling outside the LSPR, the dipolar charge accumulation on the outer rim as well as on the nanocrescent tip is strengthened. Accordingly, there are strong lobes on the outer rim of the ring and strongly/intermediately asymmetrically arranged two lobes on the tips of the nanocrescents in $0^{\circ} / 16^{\circ}$ azimuthal orientation (Fig. $8 \mathrm{~b}$, bottom).

\section{Enhancement of Dipolar Emitters via Rectangular Patterns of Different Nano-objects}

When single dipole is deepened into the convex nanoring that compose a $300-\mathrm{nm}$ rectangular pattern, all radiative rate enhancement spectra computed close to/in $\mathrm{C}$ and $\mathrm{U}$ orientation of quadrumer nanocrescents exhibit a r-PPR related shoulder $(540 \mathrm{~nm})$, and $\mathrm{r}-\mathrm{C}$ and $\mathrm{r}-\mathrm{U}$ mode related global maximum $(600 \mathrm{~nm}$ and $610 \mathrm{~nm})$, which are redshifted by $10 \mathrm{~nm}$ with respect to their counterparts on the absorptance spectra of plane wave illuminated pattern of nanorings, except the global maximum in $\mathrm{U}$ orientation that is red-shifted by $20 \mathrm{~nm}$ (Fig. 9a).

When four dipoles are arrayed in the close proximity of four slightly rotated nanocrescents that compose a quadrumer the local maxima revealing the $\mathrm{c}-\mathrm{C} 2$ and $\mathrm{c}-\mathrm{C} 1$ resonance close to / in $\mathrm{C}$ orientation appear at locations, 
(a)

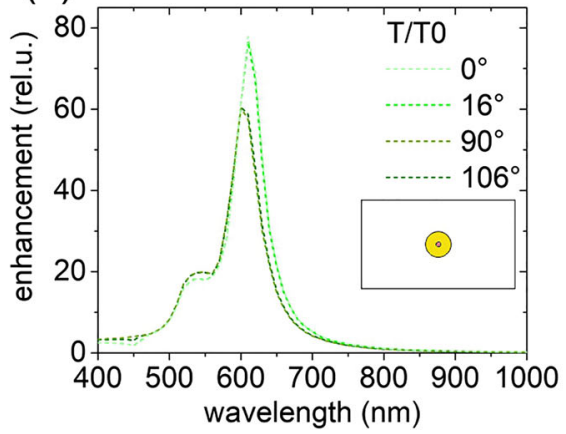

(b)

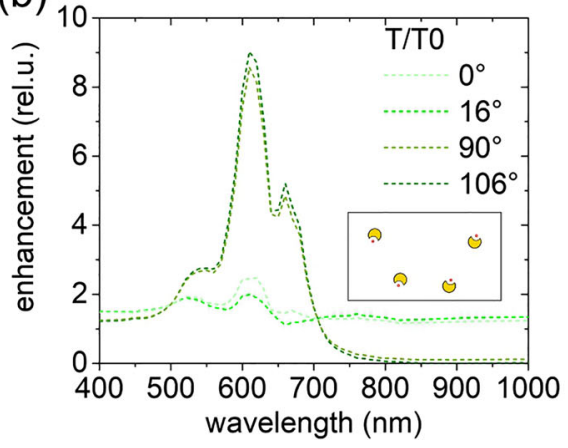

(c)

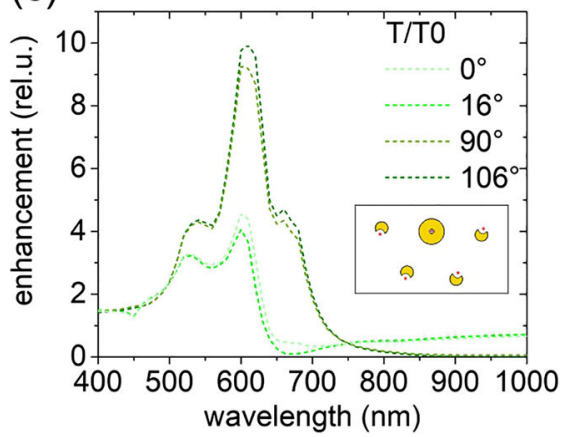

Fig. 9 Radiative rate enhancement spectra of dipolar emitters achievable via 300 nm rectangular patterns consisted of convex a nanorings, b quadrumer of nanocrescents, c complex miniarray

which are coincident $(550 \mathrm{~nm})$ and red-shifted by 10 $\mathrm{nm}(660 \mathrm{~nm})$ with respect to counterpart extrema on the absorptance spectra of plane wave illuminated quadrumers, respectively. An important difference is that a global maximum appears instead of the shoulder at a $20-\mathrm{nm}$ redshifted location $(610 \mathrm{~nm})$, which originates from a unique charge hybridization. Namely, when dipoles oscillate in close proximity of the nanocrescents, reversed strongly localized and more extended dipoles are coincident on their tips (not shown). In U orientation, c-PPR $(520 \mathrm{~nm})$ and c$\mathrm{U}$ resonance $(610 \mathrm{~nm})$ related local and global maximum appears, which is blue- and red- shifted by $10 \mathrm{~nm}$ with respect to counterpart extrema on the absorptance spectra of the plane wave illuminated analogue pattern, respectively (Fig. 9b).

In the case of the rectangular pattern composed of a complex miniarray, the peaks on the enhancement spectra are added but almost inherit the shape of the ring and quadrumer spectra, which reveals that a weak interaction occurs between the constituent convex nanoobjects. Namely, local maxima appear at spectral interval corresponding to the $\mathrm{c}-\mathrm{C} 2(540 \mathrm{~nm})$ and $\mathrm{c}-\mathrm{C} 1(660 \mathrm{~nm})$ resonance on the quadrumer, whereas in between them a large global maximum appears $(610 \mathrm{~nm})$ which originates from the mixed c-C2 and c-C1 modes overlapped with the $\mathrm{r}-\mathrm{C}$ resonance on the nanoring. These extrema are shifted by $-10 / 10 / 10 \mathrm{~nm}$ with respect to counterpart extrema on the spectra of plane wave illuminated miniarrays, respectively. In $U$ orientation, the local maximum $(530 \mathrm{~nm})$ originates from the r-PPR and c-PPR, whereas the global maximum is resulted from the $\mathrm{r}-\mathrm{U}$ and $\mathrm{c}-\mathrm{U}$ resonance on the nanorings and on the quadrumer of nanocrescents $(600 \mathrm{~nm})$. The former is coincident, whereas the latter is blue-shifted by $10 \mathrm{~nm}$ with respect to counterpart extrema on plane wave illuminated miniarrays (Fig. 9c).

\section{Discussion and Conclusion}

The hexagonal pattern of nanorings and nanocrescents has been inspected in $90^{\circ}$ and $0^{\circ}$ azimuthal orientations in order to uncover the characteristic LSPRs supported by the nano-objects. In the case of rectangular patterns, both the $90^{\circ} / 106^{\circ}$ and $0^{\circ} / 16^{\circ}$ azimuthal orientations have been studied, which are capable of promoting LSPR and gratingcoupling simultaneously/LSPR separately, depending on the unit cell composition. The common difference between the near-field and charge distributions is the clockwise rotation in $106^{\circ}$ and $16^{\circ}$ azimuthal orientation with respect to those observable at $90^{\circ}$ and $0^{\circ}$ azimuthal angles.

On the rectangular pattern of singlet convex nanorings, only the clockwise rotation is observable. On the rectangular pattern of horizontal singlet convex nanocrescents at the shoulder, hexapolar charge distribution is observable in $\mathrm{C}$ orientation $\left(90^{\circ}\right.$ azimuthal angle), whereas the charge distribution is quadrupolar close to $\mathrm{C}$ orientation (106 ${ }^{\circ}$ azimuthal angle). Symmetrical/asymmetrical dipoles develop in/close to $\mathrm{U}$ orientation $\left(0^{\circ} / 16^{\circ}\right.$ azimuthal angle $)$ at the global maximum.

On the rectangular pattern of quadrumers at the shoulder close to $\mathrm{C}$ orientation ( $90^{\circ}$ azimuthal angle), the charge distribution is more quadrupolar, whereas the charge distribution is purely hexapolar in $\mathrm{C}$ orientation $\left(106^{\circ}\right.$ azimuthal angle). Close to/in $\mathrm{U}$ orientation $\left(0^{\circ} / 16^{\circ}\right.$ azimuthal angle), the dipolar charge distribution is asymmetrical/symmetrical on each constituent nanocrescents at the global maximum.

The perfect $\mathrm{C}$ and $\mathrm{U}$ resonance arises in $90^{\circ}\left(106^{\circ}\right)$ and $0^{\circ}\left(16^{\circ}\right)$ azimuthal orientation on the rectangular pattern of singlets (quadrumers), which is capable of resulting in perfect alignment of the local $\bar{E}$-field perpendicularly and along the symmetry axes of the horizontal singlet (slightly rotated quadrumer of) nanocrescents. This explains, why 
more well-defined hexapoles arise at the shoulder in $90^{\circ}$ $\left(106^{\circ}\right)$ azimuthal orientation. The well-aligned dipoles development on the singlet (quadrumer) nanocrescents in $0^{\circ}$ $\left(16^{\circ}\right)$ azimuthal orientation can be explained by the matched orientation of their symmetry axis with the $\bar{E}$-field direction as well.

The 300-nm periodic rectangular pattern composed of a convex miniarray inherits the features of the rectangular patterns composed of a singlet nanoring and a quadrumer of nanocrescents. Due to the perfect alignment of the $\bar{E}$-field perpendicularly to the symmetry axes of the nanocrescents in $\mathrm{C}$ orientation $\left(106^{\circ}\right.$ azimuthal angle), the charge and field distribution is dominant on the nanoring at the global maximum and on the nanocrescent at the local maximum complementary. Due to the perfect alignment of the $\bar{E}$ field parallel to the symmetry axes of the nanocrescents, the sub-sets of nanocrescents are almost indistinguishable in $\mathrm{U}$ orientation ( $16^{\circ}$ azimuthal angle). In contrast to this, caused by the non-perfect perpendicularity of the $\bar{E}$-field direction to the symmetry axes, the modes on the nanoobjects extraordinarily interact close to $\mathrm{C}$ orientation (in $90^{\circ}$ azimuthal angle), as a consequence, an additional weak charge and field accumulation are observable on the nanocrescents at the global maximum and on the nanoring at the local maximum as well. Caused by the non-perfect parallelism of the $\bar{E}$-field direction to the symmetry axes close to $\mathrm{U}$ orientation $\left(0^{\circ}\right.$ azimuthal angle), the sub-sets of nanocrescents are distinguishable.

The 600-nm periodic rectangular pattern composed of a convex miniarray exhibits similar features as the 300$\mathrm{nm}$ periodic pattern. The differences between the perfect and non-perfect relative orientations are slightly less well defined, which can be explained by the smaller surface fraction of the nano-objects in the unit cell. Additional phenomenon is that the coincidence of the (almost) perfect alignment of the $\bar{E}$-field along the nanocrescents symmetry axes in $\left(0^{\circ}\right) 16^{\circ}$ azimuthal orientation and the (satisfaction) approximation of the first $( \pm 1,0)$ order grating-coupling condition of photonic modes through $\bar{k}_{p}$ lattice vector results in Rayleigh anomaly outside the spectral interval of LSPRs.

The dispersion characteristics have been analyzed in $0^{\circ}$ and $90^{\circ}$ azimuthal orientations, since these make it possible to find bands associated with coupling via grating vectors of the rectangular lattice. Only coupling via $\bar{k}_{p}$ results in welldefined bands in the inspected wavelength interval caused by the small $t$ common side length of the inspected unit cells.

The common photonic band, which interacts with the inspected localized modes at large polar angles originates from $(-1,0)$ and $(-2,0)$ order grating-coupling on the $300 \mathrm{~nm}$ and $600 \mathrm{~nm}$ periodic rectangular patterns in $90^{\circ}$ azimuthal orientation; however, this causes perturbation outside the spectral interval of LSPRs at perpendicular incidence (Fig. 4-8/c, left).

The dispersion characteristics of the hexagonal and rectangular pattern of convex nanorings do not possess azimuthal orientation dependence. A well-defined and tilting independent flat band is identifiable, which corresponds to the identical $\mathrm{r}-\mathrm{C}$ and $\mathrm{r}-\mathrm{U}$ localized plasmon resonance on the nanorings both in $90^{\circ}$ and $0^{\circ}$ azimuthal orientations (Fig. 3d, Fig. 4c).

The dispersion characteristics of the hexagonal pattern of horizontal convex nanocrescents, $300-\mathrm{nm}$ periodic rectangular pattern of horizontal singlet convex nanocrescents, and quadrumer of slightly rotated convex nanocrescents, as well as the $300 \mathrm{~nm}$ and $600 \mathrm{~nm}$ rectangular pattern of the complex miniarray exhibit a well-defined azimuthal orientation dependence (Fig. 3e, Fig. 5-8/c).

In $90^{\circ}$ azimuthal orientation of the hexagonal pattern as well as of the rectangular pattern a singlet and a quadrumer of nanocrescents, a tilting-independent weak flat band originating from the overlapping c-PPR and c-C2 and a strong flat band originating from the c-C1 LSPR appears, whereas the $\mathrm{c}-\mathrm{C} 2$ and $\mathrm{c}-\mathrm{C} 1$ modes interaction results in a ghost flat band in between them (Fig. 3e, Figs. 5-6/c left). This interaction-related band is less well defined in case of a horizontal singlet nanocrescent in a rectangular pattern (Fig. 5c left).

The dispersion characteristics of the 300-nm and 600$\mathrm{nm}$ periodic rectangular patterns of miniarrays are more complex (Fig. 7-8/c left). Similarly to the constituent nanoobjects, in $90^{\circ}$ azimuthal orientation of the 300-nm and 600$\mathrm{nm}$ periodic rectangular patterns of the miniarray, tiltingindependent weak flat bands originate from the overlapping r-PPR, c-PPR, and c-C2 as well as from the c-C1 LSPR on the nanocrescents, whereas the c-C2 and c-C1 modes interaction results in a flat band, which overlaps with the tilting and azimuthal orientation independent strong band originating from the r-C localized plasmon resonance on the nanoring.

In $0^{\circ}$ azimuthal orientation of the hexagonal pattern of horizontal convex nanocrescents, rectangular pattern of a horizontal singlet nanocrescent and a quadrumer of nanocrescents (as well as on the 300-nm and 600$\mathrm{nm}$ periodic pattern of the complex miniarray) the weak flat band originating from the c-PPR (as well as r-PPR) is separated from the tilting independent strong band originating from the c-U (as well as r-U) LSPR (Fig. 3e, Fig. 5-8/c right). In addition to this, on the dispersion map of the 600-nm complex pattern, the Rayleigh anomaly related photonic band also appears outside the spectral interval of LSPRs, due to the more/less efficient scattering of photonic modes in $( \pm 1,0)$ order on the constituent nanoobjects along the $\bar{k}_{p}$ vector corresponding to their periodic rectangular pattern in $0^{\circ} / 16^{\circ}$ azimuthal orientation. 
Our present study proves that significant enhancement of dipolar emitters is achievable in spectral intervals of plasmonic resonances on complex convex patterns that are tunable by the integrated lithography.

Acknowledgements This work was supported by the National Research, Development and Innovation Office (NKFIH) "Optimized nanoplasmonics" (K116362) and European Union, co-financed by the European Social Fund. "Ultrafast physical processes in atoms, molecules, nanostructures and biological systems" (EFOP-3.6.2-162017-00005). Á. Sipos gratefully acknowledges the support of NKFIH PD-121170.

Funding Open access funding provided by University of Szeged.

Open Access This article is licensed under a Creative Commons Attribution 4.0 International License, which permits use, sharing, adaptation, distribution and reproduction in any medium or format, as long as you give appropriate credit to the original author(s) and the source, provide a link to the Creative Commons licence, and indicate if changes were made. The images or other third party material in this article are included in the article's Creative Commons licence, unless indicated otherwise in a credit line to the material. If material is not included in the article's Creative Commons licence and your intended use is not permitted by statutory regulation or exceeds the permitted use, you will need to obtain permission directly from the copyright holder. To view a copy of this licence, visit http:// creativecommonshorg/licenses/by/4.0/.

\section{References}

1. Wang W, Ramezani M, Väkeväinen AI, Törmä P, Rivas JG, Odom TW (2018) The rich photonic world of plasmonic nanoparticle arrays. Materials Today 21:303-314. https://doi.org/10.1016/j. mattod.2017.09.002

2. Guo R, Hakala TK, Törmä P (2017) Geometry dependence of surface lattice resonances in plasmonic nanoparticle arrays. Physical Review B 95:155423. https://doi.org/10.1103/PhysRevB. 95.155423

3. Yang A, Hryn AJ, Bourgeois MR, Lee W-K, Hu J, Schatz GC, Odom TW (2016) Programmable and reversible plasmon mode engineering. Proceedings of the National Academy of Sciences of the United States of America 113:14201-14206. https://doi.org/10.1073/pnas.1615281113

4. Lévêque G, Martin OJF (2006) Tunable composite nanoparticle for plasmonics. Opt Lett 31:2750-2752. https://doi.org/10.1364/ OL.31.002750

5. Bohren CF, Huffman DR (1998) Absorption and scattering of light by small particles. Wiley, Hoboken. https://doi.org/10.1002/97835 27618156

6. Jensen TR, Schatz GC, Van Duyne RP (1999) Nanosphere lithography: surface plasmon resonance spectrum of a periodic array of silver nanoparticles by ultraviolet-visible extinction spectroscopy and electrodynamic modeling. J Phys Chem B 103:2394-2401. https://doi.org/10.1021/jp984406y

7. Ruppin R (1982) Spherical and cylindrical surface polaritons in solids. In: Boardman AD (ed) Electromagnetic Surface Modes. Wiley, New York, pp 345-398

8. Oldenburg SJ, Jackson JB, Westcott SL, Halas NJ (1999) Infrared extinction properties of gold nanoshells. Appl Phys Lett 75:28972899. https://doi.org/10.1063/1.125183

9. Aizpurua J, Hanarp P, Sutherland DS, Käll M, Bryant GW, García de Abajo FJ (2003) Optical properties of gold nanorings. Phys Rev Lett 90:057401. https://doi.org/10.1103/PhysRevLett.90.057401
10. Aizpurua J, Blanco L, Hanarp P, Sutherland DS, Käll M, Bryant GW, García de Abajo FJ (2004) Light scattering in gold nanorings. Journal of Quantitative Spectroscopy and Radiative Transfer 89:11-16. https://doi.org/10.1016/j.jqsrt.2004.05.007

11. Fan JA, Wu C, Bao K, Bao J, Bardhan R, Halas NJ, Manoharan VN, Nordlander P, Shvets G, Capasso F (2010) Selfassembled plasmonic nanoparticle clusters. Science 328:11351138. https://doi.org/10.1126/science.1187949

12. Geraci G, Hopkins B, Miroshnichenko AE, Erkihun B, Neshev DN, Kivshar YS, Maier SA, Rahmani M (2016) Polarisationindependent enhanced scattering by tailoring asymmetric plasmonic systems. Nanoscale. 8:6021-6027. https://doi.org/10.1039/c6nr 00029k

13. Fan JA, Bao K, Wu C, Bao J, Bardhan R, Halas NJ, Manoharan VN, Shvets G, Nordlander P, Capasso F (2010) Fano-like interference in self-assembled plasmonic quadrumer clusters. Nano Letters 10:4680-4685. https://doi.org/10.1021/n11029732

14. Lassiter JB, Sobhani H, Fan JA, Kundu J, Capasso F, Nordlander P, Halas NJ (2010) Fano resonances in plasmonic nanoclusters: geometrical and chemical tunability. Nano Letters 10:3184-3189. https://doi.org/10.1021/nl102108u

15. Li G, Hu H, Wu L (2019) Tailoring Fano lineshapes using plasmonic nanobars for highly sensitive sensing and directional emission. Physical Chemistry Chemical Physics 21:252-259. https://doi.org/10.1039/C8CP05779F

16. Kretschmann M, Maradudin AA (2002) Band structures of two-dimensional surface-plasmon polaritonic crystals. Physical Review B - Condensed Matter and Materials Physics 66:1-8. https://doi.org/10.1103/PhysRevB.66.245408

17. Haynes CL, McFarland AD, Zhao L, Van Duyne RP, Schatz GC, Gunnarsson L, Prikulis J, Kasemo B, Käll M (2003) Nanoparticle optics: the importance of radiative dipole coupling in two-dimensional nanoparticle arrays $\uparrow$. J Phys Chem B 107:73377342. https://doi.org/10.1021/jp034234r

18. Murray WA, Astilean S, Barnes WL (2004) Transition from localized surface plasmon resonance to extended surface plasmonpolariton as metallic nanoparticles merge to form a periodic hole array. Physical Review B - Condensed Matter and Materials Physics 69:165407-1-165407-7. https://doi.org/10.1103/PhysRevB. 69.165407

19. Chu Y, Schonbrun E, Yang T, Crozier KB (2008) Experimental observation of narrow surface plasmon resonances in gold nanoparticle arrays. Appl Phys Lett 93:181108. https://doi.org/10. $1063 / 1.3012365$

20. Auguié B, Barnes WL (2008) Collective resonances in gold nanoparticle arrays. Phys Rev Lett 101:143902. https://doi.org/10. 1103/PhysRevLett.101.143902

21. Shahmansouri A, Rashidian B (2011) Comprehensive threedimensional split-field finitedifference time-domain method for analysis of periodic plasmonic nanostructures: Near- and far-field formulation. J Optical Soc Am B: Optical Phys 28:2690-2700. https://doi.org/10.1364/JOSAB.28.002690

22. Nishijima Y, Rosa L, Juodkazis S (2012) Surface plasmon resonances in periodic and random patterns of gold nano-disks for broadband light harvesting. Opt Express 20:11466-11477. https://doi.org/10.1364/OE.20.011466

23. Rochholz H, Bocchio N, Kreiter M (2007) Tuning resonances on crescent-shaped noble-metal nanoparticles. New J Phys 9:53. https://doi.org/10.1088/1367-2630/9/3/053

24. Okamoto T, Fukuta T, Sato S, Haraguchi M, Fukui M (2011) Visible near-infrared light scattering of single silver split-ring structure made by nanosphere lithography. Opt Express 19:7068. https://doi.org/10.1364/OE.19.007068

25. Gwinner MC, Koroknay E, Liwei F, Patoka P, Kandulski W, Giersig M, Giessen H (2009) Periodic large-area metallic split-ring resonator metamaterial fabrication based on shadow nanosphere 
lithography. Small. 5:400-406. https://doi.org/10.1002/smll.2008 00923

26. Zentgraf T, Meyrath TP, Seidel A, Kaiser S, Giessen H, Rockstuhl C, Lederer F (2007) Babinet's principle for optical frequency metamaterials and nanoantennas. Phys Rev B 76:033407. https://doi.org/10.1103/PhysRevB.76.033407

27. Klein MW, Enkrich C, Wegener M, Soukoulis CM, Linden S (2006) Single-slit split-ring resonators at optical frequencies: limits of size scaling. Opt Lett 31:1259-1261. https://doi.org/10. 1364/OL.31.001259

28. Lahiri B, McMeekin SG, Khokhar AZ, De La Rue RM, Johnson NP (2010) Magnetic response of split ring resonators (SRRs) at visible frequencies. Opt Express 18:3210-3218. https://doi.org/10.1364/OE.18.003210

29. Zhou J, Koschny T, Kafesaki M, Economou EN, Pendry JB, Soukoulis CM (2005) Saturation of the magnetic response of splitring resonators at optical frequencies. Phys Rev Lett 95:223902. https://doi.org/10.1103/PhysRevLett.95.223902

30. Caglayan H, Bulu I, Loncar M, Ozbay E (2009) Experimental observation of subwavelength localization using metamaterialbased cavities. Opt Lett 34:88-90. https://doi.org/10.1364/OL. 34.000088

31. Aydin K, Pryce IM, Atwater HA (2010) Symmetry breaking and strong coupling in planar optical metamaterials. Opt Express 18:13407-13417. https://doi.org/10.1364/OE.18.013407

32. Li X, Bian X, Milne WI, Chu D (2016) Fano resonance engineering in mirror-symmetry-broken $\mathrm{THz}$ metamaterials. Appl Phys B 122:95. https://doi.org/10.1007/s00340-016-6372-5

33. Hanarp P, Käll M, Sutherland DS (2003) Optical properties of short range ordered arrays of nanometer gold disks prepared by colloidal lithography. J Phys Chem B 107:5768-5772

34. Yu L, Liu GL, Kim J, Mejia YX, Lee LP (2005) Nanophotonic crescent moon structures with sharp edge for ultrasensitive biomolecular detection by local electromagnetic field enhancement effect. Nano Letters 5:119-124. https://doi.org/10.1021/ nl048232+

35. Zhou L, Ding F, Chen H, Ding W, Zhang W, Chou SY (2012) Enhancement of immunoassay's fluorescence and detection sensitivity using three-dimensional plasmonic nano-antenna-dots array. Analytical Chemistry 84:4489-4495. https://doi.org/10.1021/ac 3003215

36. Blanco LA, García De Abajo FJ (2004) Spontaneous light emission in complex nanostructures. Phys Rev B - Condensed Matter and Materials Physics 69:205414-1-205414-12. https://doi.org/10.1103/PhysRevB.69.205414

37. Yang Y, Zhen B, Hsu CW, Miller OD, Joannopoulos JD, SoljaCcić M (2016) Optically thin metallic films for highradiative-efficiency plasmonics. Nano Letters 16:4110-4117. https://doi.org/10.1021/acs.nanolett.6b00853

38. Zhu FQ, Fan D, Zhu X, Zhu J-G, Cammarata RC, Chien C-L (2004) Ultrahigh-density arrays of ferromagnetic nanorings on macroscopic areas. Advanced Materials 16:2155-2159. https://doi.org/10.1002/adma.200400675

39. Bochenkov EV, Sutherland DS (2018) Chiral plasmonic nanocrescents: large-area fabrication and optical properties. Optics Express 26:27101. https://doi.org/10.1364/OE.26.027101

40. Kosiorek A, Kandulski W, Chudzinski P, Kempa K, Giersig M (2004) Shadow nanosphere lithography: Simulation and experiment. Nano Letters 4:1359-1363. https://doi.org/10.1021/n1049361t

41. Kosiorek A, Kandulski W, Glaczynska H, Giersig M (2005) Fabrication of nanoscale rings, dots, and rods by combining shadow nanosphere lithography and annealed polystyrene nanosphere masks. Small 1:439-444. https://doi.org/10.1002/smll.200400099

42. Nemiroski A, Gonidec M, Fox JM, Jean-Remy P, Turnage E, Whitesides GM (2014) Engineering shadows to fabricate optical metasurfaces. ACS Nano 8:11061-11070. https://doi.org/10.1021/ nn504214b
43. McLellan JM, Geissler M, Xia Y (2004) Edge spreading lithography and its application to the fabrication of mesoscopic gold and silver rings. J Am Chem Soc 126:10830-10831. https://doi.org/10.1021/ja0470766

44. Geissler M, McLellan JM, Chen J, Xia Y (2005) Side-by-side patterning of multiple alkanethiolate monolayers on gold by edge-spreading lithography. Angewandte Chemie - International Edition 44:3596-3600. https://doi.org/10.1002/anie.200500421

45. Goerlitzer ESA, Mohammadi R, Nechayev S, Banzer P, Vogel N (2018) Large-Area 3D plasmonic crescents with tunable chirality. Adv Optical Mater 7:1801770. https://doi.org/10.1002/adom.2018 01770

46. Yang S-M, Jang SG, Choi D-G, Kim S, Yu HK (2006) Nanomachining by colloidal lithography. Small. 2:458-475. https://doi.org/10.1002/smll.200500390

47. Jun Y, Yu D, George MC, Braun PV (2010) Holographically defined nanoparticle placement in 3D colloidal crystals. J Am Chem Soc 132:9958-9959. https://doi.org/10.1021/ja1023628

48. Vogel N, De Viguerie L, Jonas U, Weiss CK, Landfester K (2011) Wafer-scale fabrication of ordered binary colloidal monolayers with adjustable stoichiometries. Advanced Functional Materials 21:3064-3073. https://doi.org/10.1002/adfm.201100414

49. Sipos Á, Somogyi A, Szabó G, Csete M (2014) Plasmonic spectral engineering via interferometric illumination of colloid sphere monolayers. Plasmonics. 9:1207-1219. https://doi.org/10.1007/ s11468-014-9732-1

50. Csete M, Sipos Á, Szalai A, Szabo G (2012) Theoretical study on interferometric illumination of gold colloid-sphere monolayers to produce complex structures for spectral engineering. IEEE Photonics Journal 4:1909-1921. https://doi.org/10.1109/JPHOT.2012. 2218587

51. Sipos Á, Szalai A, Csete M (2012) Integrated lithography to prepare arrays of rounded nano-objects. Alternative Lithographic Technologies IV:83232E. https://doi.org/10.1117/12.916403

52. Sipos Á, Tóth E, Török A, Fekete OA, Szabó G, Csete M (2019) Spectral engineering via complex patterns of rounded concave and convex nanoresonators achievable via integrated lithography realized by circularly polarized light. TechConnect Briefs 373-376

53. Hubert C, Rumyantseva A, Lerondel G, Grand J, Kostcheev S, Billot L, Vial A, Bachelot R, Royer P, Chang S-H, Gray SK, Wiederrecht GP (2005) Near-field photochemical imaging of noble metal nanostructures. Nano Letters 5:615-619. https://doi.org/10.1021/n1047956i

54. Wang Z, Cheng F, Winsor T, Liu Y (2016) Optical chiral metamaterials: a review of the fundamentals, fabrication methods and applications. Nanotechnology 27:412001. https://doi.org/10.1088/ 0957-4484/27/41/412001

55. Tóth E, Sipos Á, Fekete OA, Csete M Spectral engineering via complex patterns of circular nano-object miniarrays: II concave patterns tunable by integrated lithography realized by circularly polarized light

56. Fekete OA, Tóth E, Sipos Á, Csete M Comparative study on arrays of rounded convex and concave objects achievable in integrated lithography realized by circularly polarized light

57. Born M, Wolf E (1986) Principles of optics. Pergamon Press, Oxford

58. Palik ED (ed) (1998) Handbook of optical constants of solids. Acad. Press, San Diego

59. Kats MA, Yu N, Genevet P, Gaburro Z, Capasso F (2011) Effect of radiation damping on the spectral response of plasmonic components. Optics Express 19:21748-21753. https://doi.org/10.1364/ OE.19.021748

Publisher's Note Springer Nature remains neutral with regard to jurisdictional claims in published maps and institutional affiliations. 